\title{
Evaporation and condensation of water mist/cloud droplets with
}

\section{thermal radiation}

\author{
M. Q. Brewster \\ Department of Mechanical Science and Engineering \\ University of Illinois \\ Urbana, IL 61801 \\ Tel. 217-244-1628 Fax 217-333-1942 \\ brewster@illinois.edu
}

\begin{abstract}
The role of thermal radiation in water droplet evaporation and condensation is investigated theoretically. The primary droplet size regime considered is that between non-continuum gas effects and gas-liquid velocity-slip effects, nominally $10^{-1}$ to $10^{2} \mu \mathrm{m}$, with mist or clouds (nominally $20-\mu \mathrm{m}$ diameter) being the primary application of interest. Principles for extending the results to larger droplets are also discussed, even to the radiative-evaporative balance of the oceans. The importance of distributed (volumetric, in-depth) absorption in the droplet, conduction, and advection induced by surface regression are analyzed. It is found that advection within the liquid phase induced by surface regression can be neglected for most conditions and that conduction usually establishes a spatially isothermal droplet. Comparison with experimental results for a laser-irradiated droplet suggests that, due to the isothermal condition, these assumptions are reasonable for predicting evaporation rate. Moreover, it is shown that infrared absorption and emission can be treated as surface phenomena; in-depth effects can be neglected. Extending the analysis to cloud droplets shows that radiation, whether with colder upper layers of the atmosphere, warmer lower layers/ground, or solar radiation, can have a significant influence on cloud droplet growth and stability. For typical ambient conditions the thermal radiation effect near the edge of a cloud can be as strong as the effect of a $0.1 \%$ super- or sub-saturation, enough to modify significantly the Kohler curves governing equilibrium droplet size distribution and enough to provide the missing mechanism of warm rain droplet growth between the upper limit of non-radiating, diffusional condensation growth $(\sim 30 \mu \mathrm{m})$ and the lower limit of turbulent-inertial coalescence growth $(\sim 80 \mu \mathrm{m})$, the so-called condensation-coalescence "bottleneck".
\end{abstract}

Keywords: water; droplets; evaporation; condensation; radiation; clouds 


\section{Nomenclature}

Symbols

Values given are for $20-22^{\circ} \mathrm{C}$ and $86 \mathrm{kPa}$ (or $70 \mathrm{kPa}$ )

$B_{h, m} \quad$ B-number for heat or mass transfer, Eq. (11)

$\mathrm{Bi} \quad$ Biot number, $\mathrm{k} / \mathrm{k}_{\ell}$ in small droplet (vapor conduction) limit

$\mathrm{Bi}_{r} \quad$ radiative Biot number for droplet defined in Eq. (23)

C liquid water specific heat, $4.2 \mathrm{~kJ} / \mathrm{kg}-\mathrm{K}$

$\mathrm{C}_{\mathrm{p}} \quad$ vapor specific heat, $1.0 \mathrm{~kJ} / \mathrm{kg}-\mathrm{K}$

D droplet diameter

$D_{c} \quad$ characteristic droplet diameter for Kelvin effect

$D_{0} \quad$ initial droplet diameter

$f_{s} \quad$ fraction of radiation absorbed at interface

$\mathrm{F}_{\mathrm{sr}} \quad$ radiation view factor from droplet to surroundings

g gravitational constant, $9.8 \mathrm{~m} / \mathrm{s}^{2}$

$\mathrm{h}$ enthalpy

$h_{\mathrm{fg}} \quad$ latent enthalpy of evaporation, $2454 \mathrm{~kJ} / \mathrm{kg}$

$h_{r} \quad$ radiative heat transfer coefficient

$\mathrm{H}$ non-dimensional parameter in Eqs. (44-46), $\mathrm{h}_{\mathrm{fg}} / \mathrm{R}_{\mathrm{w}} \mathrm{T}^{2}$

$\mathrm{k} \quad$ vapor thermal conductivity, $2.60 \times 10^{-2} \mathrm{~W} / \mathrm{m}-\mathrm{K}$

$\mathrm{k}_{\ell} \quad$ liquid water thermal conductivity, $0.60 \mathrm{~W} / \mathrm{m}-\mathrm{K}$

$\mathrm{K}_{\mathrm{a}}$ spectral, volumetric absorption coefficient in liquid water

$\mathrm{L} \quad$ thickness of vapor phase (distance between s- and e-states)

Le Lewis number, $\alpha_{m} / \alpha_{h}=\operatorname{Pr} /$ Sc, 1.13 (1.15)

$\mathrm{m}_{1} \quad$ water mass fraction in vapor phase

$\dot{m}^{\prime \prime} \quad$ evaporating or condensing water mass flux at vapor-liquid interface

$\mathrm{Nu} \quad$ Nusselt number

$\mathrm{P} \quad$ total pressure, $86 \mathrm{kPa}(70 \mathrm{kPa})$

$P_{s} \quad$ water saturation pressure

$\mathrm{P}_{\mathrm{s}, \mathrm{e}} \quad$ saturation pressure at $\mathrm{T}_{\mathrm{e}}=20^{\circ} \mathrm{C}: 2.315 \mathrm{kPa}$

$\mathrm{P}_{1} \quad$ water vapor partial pressure

$\mathrm{Pr} \quad$ Prandtl number, $v / \alpha_{\mathrm{h}}, 0.690(0.695)$

$\mathrm{q}_{\mathrm{r}, \mathrm{c}, \mathrm{fg}}$ heat flux $(\mathrm{r}=$ radiative, $\mathrm{c}=$ conductive, $\mathrm{fg}=$ latent $)$

$Q_{a} \quad$ droplet total (surface plus volumetric) absorption efficiency

$Q_{a, s} \quad$ droplet surface absorption efficiency

$Q_{a, v}$ droplet volumetric absorption efficiency

$Q_{r} \quad$ non-dimensional parameter in Eqs. (44-46), $Q_{a} q_{r} H / 2 k$

$r$ radial coordinate in droplet

$\mathrm{R} \quad$ droplet radius

$\mathrm{Ra}$ Rayleigh number

$\mathrm{R}_{\mathrm{w}} \quad$ gas constant for water, 8.314/18 kJ/kg-K

$\mathrm{S}$ supersaturation, $\mathrm{RH}-1$ 
$S_{\text {eq }} \quad$ equilibrium supersaturation

Sc Schmidt number, $v / \alpha_{m}, 0.608(0.603)$

$t \quad$ optical depth in liquid along some path length, e.g., $K_{a} D$

$\mathrm{T}$ temperature

$\mathrm{T}_{\mathrm{r}} \quad$ effective radiation environment temperature

$\mathrm{V}$ interface regression speed, $\dot{\mathrm{m}}^{\prime \prime} / \rho_{\ell}$

$\mathrm{x} \quad$ Cartesian coordinate in liquid phase

y Cartesian coordinate in vapor phase

\section{Greek}

$\alpha_{\mathrm{h}} \quad$ vapor thermal diffusivity, $2.58 \times 10^{-5}\left(3.11 \times 10^{-5}\right) \mathrm{m}^{2} / \mathrm{s}$

$\alpha_{m} \quad$ water vapor-air mass diffusivity, $2.93 \times 10^{-5}\left(3.58 \times 10^{-5}\right) \mathrm{m}^{2} / \mathrm{s}$

$\beta_{\mathrm{r}} \quad$ non-dimensional parameter for surface absorption assumption under optically thick, non-phase-change conditions, $\left(1-\mathrm{f}_{\mathrm{s}}\right) \mathrm{q}_{\mathrm{r}} / \mathrm{k}_{\ell} \mathrm{K}_{\mathrm{a}} \Delta \mathrm{T}$

$\Gamma \quad$ non-dimensional parameter in Eqs. (44-46), $8 \mathrm{k} / \rho_{\ell} \mathrm{h}_{\mathrm{fg}}$

$\delta_{g} \quad$ thermal convective-diffusive length scale in vapor, $k / \dot{m}^{\prime \prime} C_{p}$

$\delta_{r} \quad$ radiation absorption length scale (photon mean free path), $1 / \mathrm{K}_{\mathrm{a}}$

$\delta_{\mathrm{t}} \quad$ thermal convective-diffusive length scale in liquid, $\mathrm{k}_{\ell} / \dot{\mathrm{m}}^{\prime \prime} \mathrm{C}$

$\Delta \mathrm{T}_{\max }$ maximum possible temperature rise in liquid above $\mathrm{T}_{\mathrm{s}}$ due to in-depth absorption: Eq. 6 in general (shortwave or longwave radiation); Eq. 7b or $22 \mathrm{a}$ for typical conditions with IR radiation: $\left(1-\mathrm{f}_{\mathrm{s}}\right) \mathrm{q}_{\mathrm{r}} / \mathrm{k}_{\ell} \mathrm{K}_{\mathrm{a}}$

$\Delta \mathrm{T}$ droplet surface temperature above vapor ambient, $\mathrm{T}_{\mathrm{s}}-\mathrm{T}_{\mathrm{e}}$

$v \quad$ vapor momentum diffusivity, $1.78 \times 10^{-5}\left(2.16 \times 10^{-5}\right) \mathrm{m}^{2} / \mathrm{s}$

$\rho \quad$ vapor density, $1.01(0.823) \mathrm{kg} / \mathrm{m}^{3}$

$\rho_{\ell} \quad$ water liquid density, $1000 \mathrm{~kg} / \mathrm{m}^{3}$

$\sigma \quad$ Stefan-Boltzmann constant, $5.67 \times 10^{-8} \mathrm{~W} / \mathrm{m}^{2} \mathrm{~K}^{4}$

$\tau \quad$ droplet thermal time constant, $\rho_{\ell} \mathrm{CR}^{2} / 3 \mathrm{k}$

$\tau_{\mathrm{v}} \quad$ volumetric transmissivity through droplet

$\Psi \quad$ non-dimensional parameter in Eqs. (44-46), $k R_{w} T / \alpha_{m} h_{f g} P_{s, e}$

\section{Subscripts}

1 water species

c conductive

e thermodynamic state in vapor far from liquid-vapor interface

fg saturated liquid-vapor phase change

$\ell \quad$ liquid water

$\mathrm{L} \quad$ thickness of vapor phase (distance between s- and e-states)

0 thermodynamic state in liquid far from liquid-vapor interface, or initial $\left(R_{\circ}\right)$

$r$ radiative

s thermodynamic state in vapor at liquid-vapor interface

$\mathrm{t}$ thermal 
$\mathrm{u}$ thermodynamic state in liquid at liquid-vapor interface

w water

Acronyms and Abbreviations

IR infrared

LHS left-hand side

LW longwave

NIR near infrared

QS quasi-steady

RH relative humidity, $\mathrm{P}_{1}(\mathrm{~T}) / \mathrm{P}_{\mathrm{s}}(\mathrm{T})$

RHS right-hand side

SW shortwave

VIS visible

w.r.t with respect to 


\section{Introduction}

The effects of thermal radiation on evaporation and condensation of liquid water droplets suspended in air have been studied theoretically over several decades with a variety of motivations and assumptions. Williams conducted one of the first rigorous theoretical treatments [1], with an eye toward delineating regimes of vaporization behavior by considering, for example, conduction- and radiation-dominated behavior, and materials with negligible condensation flux ("unopposed surface rate process") such as plastics, as well as materials with near equilibrium conditions between evaporation and condensation fluxes, such as water. That study, however, assumed optically thin, (uniform) volumetric radiation absorption within the droplet, which effectively limited the results to shortwave-visible (SW-VIS) and/or near-infrared (NIR) radiation for water droplets of tens of microns or larger, or smaller droplets for longwave-infrared $(\mathrm{LW}-\mathrm{IR}$ ) radiation, e.g., $\mathrm{R}<2 \mu \mathrm{m}$ for $10.6-\mu \mathrm{m} \mathrm{CO}$ laser radiation. With regard to the latter case, and with the application of high-energy laser beam propagation through atmospheres containing aerosols in mind, Davies and Brock conducted a detailed numerical study of $10.6-\mu \mathrm{m} \mathrm{CO}_{2}$ laser radiation on small droplets, $\mathrm{R}<2$ $\mu \mathrm{m}$ [2]. The results were limited to droplets with $R<2 \mu \mathrm{m}$ in order to assure uniform volumetric radiation absorption in the droplet and maintain spherical symmetry. Kelvin (surface tension) and non-continuum effects were included. A particular focus of that study was to investigate the effect of quasi-assumptions of previous studies. In an extension of the Davies and Brock study and motivated by fog clearing and cloud processes, Varghese and Gangamma examined the effect of absorbing inclusions such as carbon in droplets and included broadband solar radiation [3]. By contrast, in the present work we consider (among other things) non-uniform absorption of radiation in droplets. We also investigate the applicability of one-dimensional analysis to absorption of $10.6-\mu \mathrm{m} \mathrm{CO}$ laser radiation in large droplets by comparison with experimental laser-induced evaporation measurements.

Another application that has attracted interest is spray processes for the purposes of cooling and humidification, including fire extinguishing. These processes typically involve much larger droplets, say, $R>50 \mu \mathrm{m}$ and usually infrared (IR) radiation. Miliauskas and Sabanas studied the interaction of transfer processes during unsteady evaporation of large water droplets using geometric optics and realistic (spectral, temperature-dependent) water optical (absorption) properties (broadband), including refraction/reflection effects [4]. A similar study by Tseng and Viskanta included the effect of thermal expansion by the droplet [5].

In all of this (and more) activity, there has usually been a particular application in mind or at least a particular assumption, such as uniform volumetric absorption or spectral integration over broadband radiation, that has avoided or obscured the effect of in-depth absorption of radiation. In this paper we seek to include in-depth absorption effects while considering realistic optical properties that cover VIS, NIR, and IR ranges. To be sure, there are still 
limitations in the present analysis, as given below, and we do ultimately have a particular application in mind (cloud microphysics); however, we believe the present analysis offers some new insights into radiation effects in water droplet evaporation and condensation in a useful, generalized theoretical framework.

We consider the effect of thermal radiation on the evaporation and condensation behavior of water in air, both in bulk and as small droplets. We address theoretically the following question: how does radiation affect water temperature and mass transfer rate? We consider both longwave (LW) infrared and shortwave (SW) solar radiation effects. And we investigate the question of spatial distribution of absorption of radiation in one-dimensional systems; does treatment of surface vs. volumetric absorption/emission matter? Textbooks that focus on the heat and mass transfer on the gas side of the problem (as opposed to the liquid side, which is the focus here) are ambivalent on this question. One popular text [6] treats the surface or interface as transparent, with all the radiation taking place volumetrically or in-depth, while another [7] does the opposite, taking the interface as completely opaque. Is the choice arbitrary for water in air? We explore this question by considering realistic water optical properties (SW and LW). However, our aim is not to incorporate spectral integration over broadband radiation, which would mask the effects of volumetric absorption. Our aim is to investigate modeling assumptions of radiation in water droplet evaporation and condensation, in particular the assumption of in-depth vs. surface absorption and emission.

We also consider limiting cases of conduction- and radiation-dominated behavior and examine behavior regimes. In these respects, this work is similar in approach to Williams [1]. Unlike that work, however, we do not restrict radiation absorption to optically thin droplet conditions (and we do not consider plastics). In terms of droplet size we consider droplets as large as possible without needing to include external flow effects (up to several tens of microns; although external flow can be treated as illustrated below) and as small as possible without needing to include solution effects or non-continuum effects (down to a few microns). We start with the Cartesian problem, applicable to bulk water and large droplets-the definition of "large" depending on wavelength as discussed below, to illustrate a few basic points, and then finish with the spherical problem, applicable to a wider size range of droplets, including cloud drops.

\section{Analysis - Cartesian Problem, Large-Droplet Limit}

Consider a large droplet of water of radius $\mathrm{R}$ in air (Fig. 1a) and the vanishingly thin interface between liquid water and vapor air-water where liquidvapor phase change of water occurs (Fig. 1b). The water can be either evaporating (with mass flux $\left.\dot{m}^{\prime \prime}>0\right)$ or condensing $\left(\dot{m}^{\prime \prime}<0\right)$. The energy balance on the interface is:

$$
q_{c, u}+q_{r} f_{s}=q_{c, s}+\dot{m}^{\prime \prime} h_{f g} .
$$


The interface energy balance indicates that the heat required for evaporation $\left(\mathrm{q}_{\mathrm{fg}}=\dot{\mathrm{m}}^{\prime \prime} \mathrm{h}_{\mathrm{fg}}\right)$ can be supplied to the interface by conduction $\left(\mathrm{q}_{\mathrm{c}}\right)$ and/or radiation $\left(q_{r}\right)$. Conduction can be supplied from the liquid side $\left(q_{c, u}\right)$ or the gas side $\left(-q_{c, s}\right)$; it is usually from one or the other, but also can be supplied from both simultaneously. The net radiant flux at the surface is initially considered to be only the incident radiation from the gas side, without appreciable emission or reflection by the water. The effect of how this incident radiation is absorbed spatially by the water is one of the points investigated in this study. Classically, absorption of incident radiation requires volume; a vanishingly thin interface cannot absorb radiation, it is generally thought. This is the convention adopted in [6]. However, in mathematical modeling, the assumption of surface absorption is often made, particularly for a relatively infrared-opaque material like water where the length-scale for absorption is often much smaller than other thermal transport length-scales. This is the convention adopted in [7]. Here we explore the impact of assuming different amounts of surface (i.e., interfacial) absorption vs.

volumetric absorption. The fraction of radiation absorbed in the interface is designated as $\mathrm{f}_{\mathrm{s}}$; the fraction transmitted through the interface, available to be absorbed volumetrically, in-depth, is $1-f_{s}$. While $f_{s}$ is considered to be zero classically from a physical point of view, and treating it otherwise has only been done for infrared modeling convenience as noted above, here we consider the case of $f_{s}>0$ for one other reason: the intriguing possibility of phase-change radiation, radiation absorbed or emitted at the interface [8].

A flux sign convention is adopted as shown in Fig. 1: fluxes are positive in the $y$-direction (negative in the r-direction), except $q_{r}$. For conduction-driven evaporation (with small, possibly negligible, radiation), the sign of $\mathrm{q}_{\mathrm{c}, \mathrm{s}}$ would be negative, i.e., heat to the interface, with $T_{s}<T_{e}$. Positive gas-conductive flux $q_{c, s}$ $>0$ could occur in the case of radiation-driven evaporation with $T_{s}>T_{e}$ such that excess heat not used for evaporation is conducted to the gas (e-state). During the initial transient period following an environmental change, i.e., boundary condition change, heat for evaporation could also be drawn from the droplet (liquid side, $\mathrm{q}_{\mathrm{c}, \mathrm{u}}$ ). This would continue until the droplet reached a quasi-steady (QS) condition, which is often characterized by constant $T_{\mathrm{s}}$ but not necessarily so. The energy balance on the QS liquid phase between the o-state and u-state for the Cartesian problem (Fig. 1b) for an optically thick (opaque) liquid is:

$$
\mathrm{q}_{\mathrm{c}, \mathrm{u}}=\dot{\mathrm{m}}^{\prime \prime}\left(\mathrm{h}_{\mathrm{o}}-\mathrm{h}_{\mathrm{u}}\right)+\left(1-\mathrm{f}_{\mathrm{s}}\right) \mathrm{q}_{\mathrm{r}} .
$$

For adiabatic evaporation (adiabatic liquid), an assumption of zero net heat flux means $q_{c, u}=\left(1-f_{s}\right) q_{r}$, and the QS energy balance gives $h_{o}=h_{u}$ or $T_{o}=T_{s}$. Note that "adiabatic liquid" does not mean zero radiant flux; it means that all the radiant energy absorbed volumetrically, in-depth, is returned to the interface via conduction. Below we also consider the non-evaporating or non-advecting limit, $\dot{m}^{\prime \prime} \rightarrow 0$, in which case the QS energy balance gives $q_{c, u}=\left(1-f_{s}\right) q_{r}$ without $T_{o}$ and $T_{s}$ necessarily being equal. 


\subsection{Volumetric vs. Surface Absorption: Liquid-Phase Equations}

For large enough droplets, curvature of the interface can be ignored. The water can be modeled as an opaque, semi-infinite slab with a moving boundary, subject to a collimated, monochromatic incident radiant flux, of which the net absorbed part is $\mathrm{q}_{\mathrm{r}}$ (surface reflection loss having already been removed or assumed to be negligible). Thermal emission by the water is neglected initially. For QS (but not necessarily adiabatic) conditions in the water (no storage term) the differential energy equation and boundary conditions are:

$$
\begin{aligned}
& \mathrm{k}_{\ell} \frac{\mathrm{d}^{2} \mathrm{~T}}{\mathrm{dx^{2 }}}+\dot{\mathrm{m}}^{\prime \prime} \mathrm{C} \frac{\mathrm{dT}}{\mathrm{dx}}+\left(1-\mathrm{f}_{\mathrm{s}}\right) \mathrm{q}_{\mathrm{r}} \mathrm{K}_{\mathrm{a}} \mathrm{e}^{-\mathrm{k}_{\mathrm{a}} \mathrm{x}}=0 \\
& \left.\mathrm{~T}\right|_{\mathrm{x}=0}=\mathrm{T}_{\mathrm{s}} ;\left.\quad \mathrm{T}\right|_{\mathrm{x} \rightarrow \infty}=\mathrm{T}_{0} .
\end{aligned}
$$

The spatial distribution of in-depth absorption of $\left(1-f_{s}\right) q_{r}$ is set by the absorption coefficient, $K_{a}$, the inverse of which defines the mean free photon path or characteristic length scale for transport of radiation in the liquid, $\delta_{r}$. The evaporation rate and surface temperature are assumed known at this point. Later, they are solved for when the gas-phase equations are added to form the coupled problem. The solution of the liquid problem is:

$$
\begin{aligned}
& \mathrm{T}_{\mathrm{s}} \neq \mathrm{T}_{\mathrm{o}}: \frac{\mathrm{T}(\mathrm{x})-\mathrm{T}_{\mathrm{o}}}{\mathrm{T}_{\mathrm{s}}-\mathrm{T}_{\mathrm{o}}}=\left[1+\frac{\Delta \mathrm{T}_{\max }}{\mathrm{T}_{\mathrm{s}}-\mathrm{T}_{\mathrm{o}}}\right] \mathrm{e}^{-\mathrm{x} / \delta_{\mathrm{t}}}-\left[\frac{\Delta \mathrm{T}_{\max }}{\mathrm{T}_{\mathrm{s}}-\mathrm{T}_{\mathrm{o}}}\right] \mathrm{e}^{-\mathrm{x} / \delta_{\mathrm{r}}} \\
& \mathrm{T}_{\mathrm{s}}=\mathrm{T}_{\mathrm{o}}: \frac{\mathrm{T}(\mathrm{x})-\mathrm{T}_{\mathrm{s}}}{\Delta \mathrm{T}_{\max }}=\mathrm{e}^{-\mathrm{x} / \delta_{\mathrm{t}}}-\mathrm{e}^{-\mathrm{x} / \delta_{\mathrm{r}}} \\
& \Delta \mathrm{T}_{\max }=\frac{\left(1-\mathrm{f}_{\mathrm{s}}\right) \mathrm{q}_{\mathrm{r}}}{\mathrm{k}_{\ell}\left(\frac{1}{\delta_{\mathrm{r}}}-\frac{1}{\delta_{\mathrm{t}}}\right)} ; \delta_{\mathrm{r}}=\frac{1}{\mathrm{~K}_{\mathrm{a}}} ; \quad \delta_{\mathrm{t}}=\frac{\mathrm{k}_{\ell}}{\dot{\mathrm{m}}^{\prime \prime} \mathrm{C}}=\frac{\mathrm{k}_{\ell}}{\rho_{\ell} \mathrm{CV}} .
\end{aligned}
$$

Note that the general solution, Eqns. (4-6), does not necessarily assume adiabatic $\left(T_{0}=T_{s}\right)$ liquid.

The character of this solution depends strongly on the absorption coefficient of water, which is shown in Fig. 2 over the VIS through IR spectrum [9]. The largest value of absorption coefficient, $1 \mu \mathrm{m}^{-1}$, occurs at approximately 3$\mu \mathrm{m}$ wavelength and is due to strong absorption by $\mathrm{O}-\mathrm{H}$ stretching. This $\mathrm{K}_{\mathrm{a}}$ value corresponding to $\delta_{\mathrm{t}} \sim 1 \mu \mathrm{m}$. Below $3 \mu \mathrm{m}$ wavelength, absorption weakens $\left(\mathrm{K}_{\mathrm{a}}\right.$ decreases) and $\delta_{t}$ increases dramatically, reaching tens of centimeters in the NIR and a maximum of several meters in the VIS region. Over the mid-IR region (5- 
$10 \mu \mathrm{m}$ ), absorption is relatively strong primarily due to $\mathrm{H}-\mathrm{O}-\mathrm{H}$ bending, with $\delta_{\mathrm{t}}$ fluctuating around $20 \mu \mathrm{m}$.

The character of the liquid temperature solution also depends on the convective-diffusive length scale $\delta_{\mathrm{t}}$, which depends on the evaporating surface regression speed $\mathrm{V}=\dot{\mathrm{m}}^{\prime \prime} / \rho_{\ell}$. For example, if water at nominally atmospheric pressure ( $86 \mathrm{kPa}$, to be precise) and room temperature were evaporating adiabatically $\left(T_{s}=T_{0}=22^{\circ} \mathrm{C}\right)$ under the influence of an absorbed radiant flux the same order as direct solar radiation $\left(q_{r}=1070 \mathrm{~W} / \mathrm{m}^{2}\right)$ with no conductive heat flux from the gas phase $\left(q_{c, s}=0\right)$, the evaporation rate would be $V=0.43 \mu \mathrm{m} / \mathrm{s}$, giving $\delta_{\mathrm{t}}=32.7 \mathrm{~cm}$. This value of $\delta_{\mathrm{t}}(32.7 \mathrm{~cm})$ is next used in Figs. 3 and 4 as a benchmark value to illustrate various features of liquid temperature profile during evaporation with in-depth absorption, including the effect of absorption lengthscale $\delta_{\mathrm{r}}$ (additional gas-phase conditions that could give rise to this situation are discussed later with Figs. 5 and 6 ).

Figure 3 shows how, for a fixed $\delta_{\mathrm{t}}(32.7 \mathrm{~cm})$, the liquid temperature solution (solid lines) exhibits a local maximum at some $x$-location below the surface with the location depending on $\delta_{\mathrm{r}}$. For example, at the NIR wavelength of $1 \mu \mathrm{m}, \delta_{\mathrm{r}}$ is approximately $10 \mathrm{~cm}$, which is the same order as $\delta_{\mathrm{t}}(32.7 \mathrm{~cm})$. Figure 3 shows how at this wavelength the liquid temperature rises to $128^{\circ} \mathrm{C}$, or $106^{\circ} \mathrm{C}$ above the surface/bulk temperature, reaching this maximum at $0.17 \mathrm{~m}$ from the surface. (Of course, a superheat temperature as high as $128^{\circ} \mathrm{C}$ is generally not achievable in bulk water at nominally atmospheric pressure and these results should be viewed as theoretical. More realistic considerations are discussed below.) From Eq. (5) it can be seen that the location of the rise/fall of $T(x)$ is determined by the smaller/larger of $\delta_{\mathrm{r}}$ and $\delta_{\mathrm{t}}$. The value of the maximum temperature reached is related to $\Delta T_{\max }$, which is determined by $\left(1-f_{s}\right) q_{r}$ in addition to $\delta_{\mathrm{t}}$ and $\delta_{\mathrm{r}}$ (again, primarily the smaller of these two length scales), as indicated by Eq. (6). The interpretation of $\Delta \mathrm{T}_{\max }$ is the maximum temperature rise possible in the liquid above $T_{s}$; this temperature rise is achieved when one length scale, either $\delta_{\mathrm{t}}$ or $\delta_{\mathrm{r}}$, is much bigger than the other, as illustrated in the next case.

For mid-IR wavelengths $(5-10 \mu \mathrm{m}), \delta_{r}$ is approximately $20 \mu \mathrm{m}$ and for longer IR wavelengths $(>10 \mu \mathrm{m}) \delta_{\mathrm{r}}$ is even smaller, approximately $10 \mu \mathrm{m}$ [9], which is typically much less than $\delta_{\mathrm{t}}$. For the mid-IR case $\left(\delta_{\mathrm{r}}=20 \mu \mathrm{m}, \delta_{\mathrm{t}}=32.7\right.$ $\mathrm{cm}$ ), shown in detail in Fig. 4, Eq. (6) for $\Delta \mathrm{T}_{\max }$ dictates that the liquid temperature rises only $0.036^{\circ} \mathrm{C}$ above the surface/bulk temperature, and does so within $\sim 100 \mu \mathrm{m}$ of the surface. Thereafter, temperature stays constant at $22.036^{\circ} \mathrm{C}$ for several hundred $\mu \mathrm{m}$. The important points are that, at very opaque wavelengths (e.g., infrared) and for typical evaporation rates, the condition $\delta_{t}>>$ $\delta_{r}$ holds, the maximum liquid temperature achieved is approximately $T_{s}+\Delta T_{\max }$ $\left(22.036^{\circ} \mathrm{C}\right.$ in Fig. 4) with $\left|\Delta \mathrm{T}_{\max }\right|<<\left|\mathrm{T}_{\mathrm{s}}\right|$, and therefore, the liquid is essentially isothermal.

Another feature of infrared-assisted evaporation $\left(\delta_{\mathrm{t}}>>\delta_{\mathrm{r}}\right)$ is that in the region in the liquid closest to the interface $x<\delta_{t}$, (which is the entire $x$-axis in Fig. 4 up to $x=1000 \mu \mathrm{m}$ and beyond), the advective term in Eq. (3a) is negligible. This can be seen by dropping the advective term from the differential 
equation and modifying the far-field boundary condition to a zero-gradient condition with unspecified temperature ( $T_{0}$ is no longer specified but solved for):

$$
\mathrm{k}_{\ell} \frac{\mathrm{d}}{\mathrm{dx}}\left(\frac{\mathrm{dT}}{\mathrm{dx}}\right)-\left(1-\mathrm{f}_{\mathrm{s}}\right) \mathrm{q}_{\mathrm{r}} \frac{\mathrm{d}}{\mathrm{dx}}\left(\mathrm{e}^{-\mathrm{K}_{\mathrm{a}} \mathrm{x}}\right)=0 ;\left.\quad \mathrm{T}\right|_{\mathrm{x}=0}=\mathrm{T}_{\mathrm{s}} ;\left.\quad \frac{\mathrm{dT}}{\mathrm{dx}}\right|_{\mathrm{x} \rightarrow \infty}=0 .
$$

The solution to this equation (which can also be obtained from the general solution, Eqns. (4) and (6), by letting $\delta_{t} \rightarrow \infty$ ) is:

$$
\delta_{\mathrm{t}}>\delta_{\mathrm{r}}: \frac{\mathrm{T}(\mathrm{x})-\mathrm{T}_{\mathrm{s}}}{\Delta \mathrm{T}_{\max }}=1-\mathrm{e}^{-\mathrm{K}_{\mathrm{a}} \mathrm{x}} ; \Delta \mathrm{T}_{\max }=\frac{\left(1-\mathrm{f}_{\mathrm{s}}\right) \mathrm{q}_{\mathrm{r}}}{\mathrm{k}_{\ell} \mathrm{K}_{\mathrm{a}}} .
$$

This approximate solution is also plotted in Figs. 3 and 4 (dashed lines). Note how for the infrared case in Fig. $4\left(\delta_{\mathrm{t}}>>\delta_{\mathrm{r}}\right)$ the dashed and solid lines are indistinguishable over $0<\mathrm{x}<1000 \mu \mathrm{m}\left(<<32.7 \mathrm{~cm}=\delta_{\mathrm{t}}\right)$. If this were a droplet with $R=1000 \mu \mathrm{m}$ evaporating adiabatically, the non-advective solution would be perfectly adequate and the zero-gradient boundary condition would be the natural symmetry condition at $r=0$. Note also how in Fig. 3 the non-advective model is not a good one for the cases where $\delta_{r}$ is of the same order as $\delta_{t}$ (except at $x<<\delta_{r}$ ); nevertheless the non-advective solution does at least give a way of interpreting the meaning of $\Delta T_{\max }$. This insight about IR-assisted evaporation in the Cartesian problem is used below for setting up the droplet problem with IR radiation.

Finally, for the special (and unlikely) case of equal radiant and conductiveconvective length scales $\left(\delta_{t}=\delta_{r}=32.7 \mathrm{~cm}\right.$ in Fig. 3 ) the solution of Eq. (3a) for $\mathrm{T}_{\mathrm{s}}$ $=T_{0}$ is as follows.

$$
\mathrm{T}_{\mathrm{s}}=\mathrm{T}_{\mathrm{o}} ; \delta_{\mathrm{r}}=\delta_{\mathrm{t}}=\delta: \frac{\mathrm{T}(\mathrm{x})-\mathrm{T}_{\mathrm{s}}}{\frac{\left(1-\mathrm{f}_{\mathrm{s}}\right) \mathrm{q}_{\mathrm{r}}}{\mathrm{k}_{\ell}\left(\frac{1}{\delta}\right)}}=(\mathrm{x} / \delta) \mathrm{e}^{-\mathrm{x} / \delta}
$$

This value of $\delta_{\mathrm{r}}$ corresponds approximately to the wavelength range $0.7-0.8 \mu \mathrm{m}$ (VIS to NIR). At this condition $\left(\delta_{\mathrm{r}}=\delta_{\mathrm{t}}=\delta\right)$ the maximum temperature occurs at $\mathrm{x}=$ $\delta$.

An important feature of the liquid temperature profiles in Figs. 3 and 4 is that in spite of their significant differences in terms of location and magnitude of maximum temperature, all the profiles in Figs. 3 and 4 have the same slope at the surface. This has been somewhat artificially imposed by assuming constant surface temperature and constant regression speed or mass flux, and therefore constant heat flux conducted to the interface from the liquid. The gas-phase part of the problem has not been included yet (in fact, the gas-problem has so far been removed by choosing conditions so that the gas-side conductive flux is 
zero). To solve the more general coupled problem including both gas and liquid temperatures as well as evaporation rate from more basic environmental parameters we must include the gas-phase equations and the interface matching equations.

\subsection{Vapor-Phase and Interface Equations}

The differential equations for conservation of mass and energy in the vapor phase are presented in heat and mass transfer texts [6,7]. These differential equations along with matching conditions at the vapor-liquid interface can be solved using the usual choice of assumptions (Lewis number unity, Le 1; nearly equal water-vapor and air enthalpies; or "low" (diffusiondominated/dilute) mass-transfer) to give the following algebraic results:

$$
\begin{aligned}
& q_{c, s}=\overbrace{N u_{L}}^{1} \frac{k}{L}\left(T_{s}-T_{e}\right) \\
& \dot{\mathrm{m}}^{\prime \prime}=\frac{\rho \alpha_{\mathrm{m}, \mathrm{h}}}{\mathrm{L}} \ln \left(1+\mathrm{B}_{\mathrm{m}, \mathrm{h}}\right) \\
& B_{h}=\frac{h_{e}-h_{s}}{h_{s}-\left(h_{u}+\frac{q_{c, u}}{\dot{m}^{\prime \prime}}+\frac{f_{s} q_{r}}{\dot{m}^{\prime \prime}}\right)} ; \quad B_{m}=\frac{m_{1, s}-m_{1, e}}{1-m_{1, s}} \\
& \frac{h(y)-h_{s}}{h_{e}-h_{s}}\left(\approx \frac{T(y)-T_{s}}{T_{e}-T_{s}}\right)=\frac{1-\exp \left(y / \delta_{g}\right)}{1-\exp \left(L / \delta_{g}\right)} \\
& \delta_{\mathrm{g}}=\frac{\mathrm{k}}{\dot{\mathrm{m}}^{\prime \prime} \mathrm{C}_{\mathrm{p}}}=\frac{\mathrm{k}}{\rho_{\ell} \mathrm{C}_{\mathrm{p}} \mathrm{V}}
\end{aligned}
$$

Here $m_{1}$ is water-vapor mass fraction, $h(y)$ and $h_{e, s}$ are gas (dry air plus watervapor) enthalpy, $h_{u}$ is liquid enthalpy, $\alpha_{m, h}$ is vapor-phase mass or energy diffusivity (binary diffusion coefficient or thermal diffusivity), $L$ is the thickness of the vapor phase (the distance between the s-surface and the e-state), and $\delta_{\mathrm{g}}$ is the gas diffusive-convective length scale. These results, coupled with the algebraic solutions from the liquid phase, close the system mathematically. Specified inputs would typically include e-state conditions, o-state, and $\mathrm{q}_{\mathrm{r}}$. Unknowns to be solved for would include $\dot{\mathrm{m}}^{\prime \prime}, \mathrm{T}_{\mathrm{s}}$ and various heat fluxes. With the QS liquid-phase energy balance, Eq. (2), $B_{h}$ becomes independent of $f_{s}$ 


$$
B_{h}=\frac{h_{e}-h_{s}}{h_{s}-\left(h_{o}+\frac{q_{r}}{\dot{m}^{\prime \prime}}\right)}
$$

as do $B_{m}, \dot{m}^{\prime \prime}, T_{s}$, and all of the vapor-phase quantities. This explains why opposite assumptions about $f_{s}$, i.e., $f_{s}=0$ [6] and $f_{s}=1$ [7], can have no effect on the mass transfer rate or vapor quantities. Only the liquid temperature profile is a function of $\mathrm{f}_{\mathrm{s}}$, and the importance of this parameter is investigated below.

\section{Results - Cartesian Problem, Large-Droplet Limit}

Next we consider results for a numerical example, including both gas- and liquid-phase properties, to illustrate some basic features of radiation-assisted evaporation of water. For the gas phase we assume a gas-layer thickness (distance between s-state and e-state) of $L=1 \mathrm{~mm}$ (this dimension becomes droplet radius when the problem is changed to spherical coordinates). The estate is assumed to be $T_{e}=22^{\circ} \mathrm{C}, 25 \%$ relative humidity, and total pressure 86 $\mathrm{kPa}$ (elevation $1280 \mathrm{~m}$; the reason for this selection is later explained in relation to experimental comparison). For the o-state it is feasible, at least for the Cartesian problem, to consider water being fed into the system from a physical ostate at an arbitrary feedwater temperature (this is not usually feasible for the droplet problem; energy balance conditions for the droplet problem are considered separately below). Another option is to assume an adiabatic (and $\mathrm{QS}$ ) liquid, $T_{0}=T_{s}$. Since adiabatic and $Q S$ liquid is the normal condition for droplets, and we wish to use the Cartesian problem to demonstrate trends and justify key assumptions that can be used in the spherical droplet problem, we will consider that condition here also for the slab geometry. (If we were to compare the case of prescribed o-state temperature, say, $T_{0}=T_{e}$, with the adiabatic liquid case we'd find that trends don't change much anyway. Note again that "adiabatic" liquid here refers to zero heat flux to/from the bulk liquid, not the interface.)

Gas properties are taken for dilute air/water-vapor accounting for both pressure and temperature dependence. Temperature and pressure variations are accounted for by the ideal gas equation-of-state (through gas density) and the following correlation for binary diffusion coefficient.

$$
\alpha_{m}\left[\mathrm{~m}^{2} / \mathrm{s}\right]=1.97 \mathrm{e}-5\left(\frac{\mathrm{T}[\mathrm{K}]}{256}\right)^{1.685}\left(\frac{101.3}{\mathrm{P}[\mathrm{kPa}]}\right)
$$

From these two equations (ideal gas and diffusion coefficient), temperature- and pressure-dependence are transmitted to other transport properties via assumed constant Pr, Sc, and Le. (This is simply for convenience and is appropriate over a limited temperature range, such as atmospheric.) At each assumed $q_{r}$ value, properties are evaluated based on $T_{\text {avg }}=\left(T_{\mathrm{s}}+T_{\mathrm{e}}\right) / 2$. The following properties for 
$\mathrm{T}_{\text {avg }}=22^{\circ} \mathrm{C}$ (and $86 \mathrm{kPa}$ ) correspond to $\mathrm{q}_{\mathrm{r}}=1070 \mathrm{~W} / \mathrm{m}^{2}: \rho=1.01 \mathrm{~kg} / \mathrm{m}^{3} ; \mathrm{k}=$ $2.60 \mathrm{e}-2 \mathrm{~W} / \mathrm{m}-\mathrm{K} ; \mathrm{C}_{\mathrm{p}}=1.0 \mathrm{~kJ} / \mathrm{kg}-\mathrm{K} ; \mathrm{v}=1.78 \mathrm{e}-5 \mathrm{~m}^{2} / \mathrm{s} ; \alpha_{\mathrm{m}}=2.93 \mathrm{e}-5 \mathrm{~m}^{2} / \mathrm{s} ; \alpha_{\mathrm{h}}=$ $2.58 \mathrm{e}-5 \mathrm{~m}^{2} / \mathrm{s} ; \mathrm{Pr}=0.69 ; \mathrm{Sc}=0.608 ; \mathrm{Le}=1.13$. Liquid water properties are the same as used previously: $\rho_{\ell}=1000 \mathrm{~kg} / \mathrm{m}^{3} ; \mathrm{k}_{\ell}=0.60 \mathrm{~W} / \mathrm{m}-\mathrm{K} ; \mathrm{C}=4.2 \mathrm{~kJ} / \mathrm{kg}-\mathrm{K}$, with no significant temperature or pressure dependence for the conditions considered here.

With the input properties prescribed above and an assumed radiant heat flux $\mathrm{q}_{\mathrm{r}}$ we can solve for evaporation mass flux $\dot{\mathrm{m}}^{\prime \prime}$ or interface regression speed $\mathrm{V}=\dot{\mathrm{m}}^{\prime \prime} / \rho_{\ell}$, the temperature fields in the liquid $\mathrm{T}(\mathrm{x})$ and gas $\mathrm{T}(\mathrm{y})$, and all the heat fluxes. Results for $T(x)$ and $T(y)$ are shown in Fig. 5; fluxes and $T_{s}$ are shown in Fig. 6. We begin with the case of no radiant flux, then positive radiant flux (radiant heating), and finally negative radiant flux (radiant cooling).

When $q_{r}=0$, the liquid temperature is essentially isothermal at the wetbulb value, $10^{\circ} \mathrm{C}$, as shown in Fig. 5 ; there is no heat conducted to or from the interface on the liquid side. The surface regresses due to evaporation at a speed of $V=0.12 \mu \mathrm{m} / \mathrm{s}$ and the heat flux to support this evaporation comes entirely by conduction from the gas with $\mathrm{q}_{\mathrm{fg}}=-\mathrm{q}_{\mathrm{c}, \mathrm{s}}=308 \mathrm{~W} / \mathrm{m}^{2}$, as shown in Fig. 6. The temperature gradient to support this heat flux can be seen in the gas-side $(y>0)$ temperature profile in Fig. 5.

As $q_{r}$ is increased above zero, the evaporation rate increases, as well as temperatures throughout the liquid and the gas regions, as illustrated by the case $\mathrm{q}_{\mathrm{r}}=1070 \mathrm{~W} / \mathrm{m}^{2}$ in Fig. 5. At $\mathrm{q}_{\mathrm{r}}=1070 \mathrm{~W} / \mathrm{m}^{2}$ the evaporation speed has increased to $V=0.43 \mu \mathrm{m} / \mathrm{s}$ (as represented by the increase in $\mathrm{q}_{\mathrm{fg}}$ in Fig. 6 ) and the surface temperature has increased to be just equal to the e-state temperature $\left(22^{\circ} \mathrm{C}\right)$, such that the gas-side conductive heat flux $\mathrm{q}_{\mathrm{c}, \mathrm{s}}$ drops to zero. At this condition the radiant flux alone supports evaporation. Now, since $q_{r}>0$, in order to determine the liquid temperature profile it is necessary to specify the liquid optical properties, $K_{a}$ and $f_{s}$. Figure 5 shows temperature profiles for both $f_{s}=1$ and 0 , with the absorption length scale set to $1 / K_{a}=10 \mathrm{~cm}$ when $\mathrm{f}_{\mathrm{s}}=0$. This latter condition is the same condition for the liquid temperature profiles shown in Fig. 3. Note that letting $\mathrm{K}_{\mathrm{a}} \rightarrow \infty$ (more quantitatively, $1 / \mathrm{K}_{\mathrm{a}}<<\delta_{\mathrm{t}}$ ) is equivalent to letting $\mathrm{f}_{\mathrm{s}}$ $\rightarrow 1$, as also illustrated by the curve $1 / K_{a}=20 \mu \mathrm{m}$ in Figs. 3 and 4 . Thus, the two liquid profiles in Fig. 5 for each flux can be interpreted as being for different wavelength regions: near infrared $(1 \mu \mathrm{m})$ for $f_{s}=0\left(1 / K_{a}=10 \mathrm{~cm}\right)$, and midinfrared $(5-10 \mu \mathrm{m})$ for $\mathrm{f}_{\mathrm{s}}=1\left(1 / \mathrm{K}_{\mathrm{a}} \rightarrow 0\right)$.

As $\mathrm{q}_{\mathrm{r}}$ increases above $1070 \mathrm{~W} / \mathrm{m}^{2}$, radiation provides more than enough heat for evaporation; the extra heat is conducted to the gas as shown by the case $\mathrm{q}_{\mathrm{r}}=5000 \mathrm{~W} / \mathrm{m}^{2}$ in Fig. 5 . At $\mathrm{q}_{\mathrm{r}}=5000 \mathrm{~W} / \mathrm{m}^{2}$ the evaporation speed increases to $1.8 \mu \mathrm{m} / \mathrm{s}$; the required latent heat is $4500 \mathrm{~W} / \mathrm{m}^{2}$ and the remaining $500 \mathrm{~W} / \mathrm{m}^{2}$ is conducted to the gas. For $f_{s}=0$, the $5000 \mathrm{~W} / \mathrm{m}^{2}$ is absorbed in depth by the liquid and then conducted to the surface via the temperature gradient in the liquid shown in Fig. 5 . For $f_{s}=1$, the $5000 \mathrm{~W} / \mathrm{m}^{2}$ is absorbed at the interface; the liquid region is isothermal.

With this more complete description of the physical conditions of Figs. 3 and 4 in mind (i.e., gas-phase included) it is instructive to return to the liquid 
temperature profiles previously described. The liquid is assumed to be adiabatic and QS such that $q_{c, u}=\left(1-f_{s}\right) q_{r}$ and $T_{s}=T_{o}\left(=22^{\circ} \mathrm{C}\right)$. The value of $q_{r}=1070$ $\mathrm{W} / \mathrm{m}^{2}$ is just enough to drive the evaporation at $0.43 \mu \mathrm{m} / \mathrm{s}$ with no assistance by conduction from the gas such that the liquid surface and bulk temperatures are the same as the gas environment temperature: $T_{s}=T_{0}=T_{e}$. Since surface absorption is assumed zero $\left(f_{s}=0\right)$ in Figs. 3 and 4 , the adiabatic, quasi-steady condition gives: $\mathrm{q}_{\mathrm{c}, \mathrm{u}}=\mathrm{q}_{\mathrm{r}}$. This is why each of the temperature profiles in Fig. 3 has the same slope at the surface, to provide the same conductive heat flux of $1070 \mathrm{~W} / \mathrm{m}^{2}$ to the surface from the liquid. For less opaque conditions, i.e., smaller (NIR) absorption coefficients (larger $\delta_{r}$ values in Fig. 3 ), the steady, onedimensional liquid temperature (in the absence of cellular or otherwise recirculative advection) must rise to higher maximum values than for larger (IR) absorption coefficients (e.g., $\delta_{r}=20 \mu \mathrm{m}$ in Fig. 4). In reality, for a body of water centimeters or tens of centimeters in depth, volumetric heating by shortwave (SW) near-infrared radiation over length scales of centimeters or tens of centimeters would generally not allow a quiescent, one-dimensional flow to be maintained over that length scale; the water would mix three-dimensionally and smooth the average temperature profile to be more like the infrared profile of Fig. 4 with the gradient concentrated near the surface.

An important application of the condition where $\mathrm{q}_{\mathrm{r}}$ is SW and of the order of $10^{2}$ to $10^{3} \mathrm{~W} / \mathrm{m}^{2}$ is the earth's oceans under the influence of solar radiation. At mid-day in the tropics without clouds the SW solar radiation approaches $10^{3}$ $\mathrm{W} / \mathrm{m}^{2}$ whereas averaging over the spherical surface of the earth (longitude and latitude) and accounting for back-scattering, mostly by clouds, reduces the average to approximately $240 \mathrm{~W} / \mathrm{m}^{2}$. The ocean's depth generally exceeds $\delta_{\mathrm{r}}$ even for visible wavelengths. It absorbs nearly all of the SW solar radiation flux before any reaches the bottom. The net LW infrared flux emitted from the ocean to the sky, which also depends on season, latitude and sky conditions, averages approximately $120 \mathrm{~W} / \mathrm{m}^{2}$ over the earth. Therefore, the net radiative flux, SW plus LW, is absorptive (not emissive) and of a sufficient amount $(240-120=120$ $\mathrm{W} / \mathrm{m}^{2}$ average) to keep the ocean surface, in a global sense, at approximately the same average temperature as the air while undergoing net evaporation. That is, the ocean surface is neither significantly cooled below the average air temperature by evaporation nor heated above the average air temperature by solar radiation (as a black, absorbing, non-volatile solid surface would be).

Of course, as noted above, the fluid mixing state in the surface layer of the ocean is not one-dimensional. Nevertheless the average temperature gradient at the surface on the liquid side is similar qualitatively to that depicted in Figs. 3 and 4 , sufficient to provide the necessary latent heat for evaporation. (The actual magnitude of the gradient in Figs. 3 and 4, is too steep to represent average ocean-atmosphere conditions. For one thing, the radiant flux of $1070 \mathrm{~W} / \mathrm{m}^{2}$ is too large for average conditions but also, instead of an effective air layer thickness of $\mathrm{L}=1 \mathrm{~mm}$, average ocean evaporation rates, which are around $0.17 \mathrm{~mm} / \mathrm{day}$, suggest an effective value of $L$ closer to $5 \mathrm{~mm}$, and therefore a more shallow gradient.) This unique condition for earth's oceans happens because of the right 
balance between radiative heating and evaporative cooling. In terms of radiative properties of water it can be further attributed to the fact that (1) water is an efficient emitter of longwave (LW) infrared radiation and (2) ocean water absorbs nearly all of solar radiation, even SW. This situation for ocean water is in contrast with cloud drops that emit LW efficiently like the ocean but don't absorb SW well because of small size; the cloud case is considered below with the droplet problem.

The analysis above shows that for infrared radiation (i.e., $\delta_{r}$ closer to tens of $\mu \mathrm{m}$ than $\mathrm{mm}$ or $\mathrm{cm}$ ) and sufficiently low fluxes and evaporation rates, which specifically means for $\mathrm{q}_{r}$ values up to about $5000 \mathrm{~W} / \mathrm{m}^{2}$, the temperature change in the liquid is negligibly small and if the water were an evaporating droplet it would be appropriate to treat absorption as a surface phenomenon $\left(f_{s}=1\right)$ and ignore temperature gradients in the droplet. Whatever internal process might occur in a droplet, be it simple bulk advection-diffusion, as analyzed here, or more complex circulation, heat transfer in liquid water is not a limiting thermal transport factor. This finding follows from simply evaluating $\Delta T_{\max }$ as defined in Eq. (6) with $\delta_{\mathrm{t}} \gg \delta_{\mathrm{r}}$. We will use this information later in the droplet energy balance to show further that transient heat storage in a droplet is usually negligible and the QS approximation is valid even when $T_{\mathrm{s}}$ changes slowly due to say, the environmental humidity or temperature changing. Since $\Delta T_{\max }$ represents the temperature rise in the water above $T_{s}$ due to in-depth absorption effects, it can be concluded that for IR radiation, under most conditions, in-depth absorption effects, and resultant non-isothermal conditions in the liquid phase are negligible. Note that these two assumptions, surface absorption and isothermal liquid, go together, as shown for the mid-IR cases of Fig. $4\left(f_{s}=0\right.$ assumed) and Fig. $5\left(\mathrm{f}_{\mathrm{s}}=1\right.$ assumed). Thus we see from this analysis that in-depth absorption of mid-IR or longer wavelength radiation in quasi-steadily evaporating (or condensing) water can be ignored. The same is true for infrared emission, if it were included; emission can also be treated as a surface or interfacial phenomenon $\left(f_{s}=1\right)$. The value of $f_{s}$ does not affect the evaporation or condensation rate or any gas-phase quantities; only the liquid-phase temperature profile is affected by the value of $f_{s}$ and even that effect is negligible for radiant flux values below $10^{4} \mathrm{~W} / \mathrm{m}^{2}$.

Next let us include infrared emission and consider the situation in Figs. 5 and 6 for radiant fluxes below zero, i.e., net emission to an environment with an effective radiative temperature $T_{r}$ that is below $T_{s}$. Recall that at $q_{r}=0$ evaporation was entirely supported by heat conducted to the interface from the vapor phase. As $\mathrm{q}_{\mathrm{r}}$ decreases below zero, $\mathrm{T}_{\mathrm{s}}, \mathrm{V}$, and $\mathrm{q}_{\mathrm{fg}}$ decrease below the values at $\mathrm{q}_{\mathrm{r}}=0: 10^{\circ} \mathrm{C}, 0.12 \mu \mathrm{m} / \mathrm{s}$, and $308 \mathrm{~W} / \mathrm{m}^{2}$, respectively, while some of the heat supplied by conduction from the gas to the interface begins to be conducted into the liquid. From there it would be radiated to the environment (still considering QS liquid). The temperature profile for this situation would be qualitatively like that for the $q_{r}=0$ curve of Fig. 5 but with a steeper gas-phase profile. The radiation occurring under these conditions (net emission) would be typically infrared dominated and the appropriate value of $f_{s}$ to consider would be $f_{s}=1$, as discussed above. If it were possible to decrease the radiant flux value 
to $-540 \mathrm{~W} / \mathrm{m}^{2}$, the surface temperature would drop to $T_{\mathrm{s}}=1{ }^{\circ} \mathrm{C}$, and the evaporation rate (and $\mathrm{q}_{\mathrm{fg}}$ ) would go to zero (see Fig. 6). At this condition all the heat conducted to the surface would be radiated to the environment; none would be available to support evaporation.

For $q_{r}$ values below $-540 \mathrm{~W} / \mathrm{m}^{2}$, the mass transfer would reverse directions $\left(\dot{\mathrm{m}}^{\prime \prime}, \mathrm{V}\right.$, and $\mathrm{q}_{\mathrm{fg}}$ would change signs from (+) to $\left.(-)\right)$ and vapor would begin condensing at the interface with the latent heat liberated being radiated to the environment along with heat conducted from the gas. This paradoxical situation shows it is possible, with low enough environment radiation temperature, to induce condensation at the interface even when heat is being conducted from the (conductively) warmer gas to the interface. This particular value of $\mathrm{q}_{\mathrm{r}}\left(-540 \mathrm{~W} / \mathrm{m}^{2}\right)$ would be difficult to achieve between any realistic droplet and environment temperatures because of the low specified relative humidity $(25 \%)$. However, for higher environmental humidity, especially near saturation, the required $\mathrm{q}_{\mathrm{r}}$ magnitude would be much lower and thus condensation would be quite feasible, particularly at the tops of clouds (see discussion of droplet cases below). Further decreasing the radiant flux to $q_{r}=-592 \mathrm{~W} / \mathrm{m}^{2}$ (again unrealistically low) would cause $\mathrm{T}_{\mathrm{s}}$ to approach $0^{\circ} \mathrm{C}$, with the possibility of freezing (although subcooling would be more likely, at least for several degrees).

It is interesting to note that the steady-state variation of $T(x)$ with $x$ is opposite (if sometimes only slightly so) of what we learn to expect based on transient analysis of the initial warm-up process (semi-infinite "solid") with the usual assumption of $f_{s}=1$. If the water was initially in thermal equilibrium with the environment and suddenly exposed to a surface radiant flux, the concentration of absorbed heat near the surface would cause the surface region to become hotter than the interior; $\mathrm{T}(\mathrm{x})$ would decrease with increasing $\mathrm{x}$. However, eventually, at steady-state, the interior region becomes warmer (slightly for infrared heating or solar heating with mixed fluid) as noted by the steady solutions above with $\mathrm{f}_{\mathrm{s}}=0$, and $T(x)$ becomes a (slightly) increasing function of $x$.

To summarize, we have demonstrated from the one-dimensional Cartesian problem that for IR-assisted evaporation or condensation of water, the advection term in the liquid phase is generally negligible. The non-advective Cartesian analysis is also applicable to large droplets with IR radiation, i.e., droplets with radius $R>>1 / K_{a} \sim 20 \mu \mathrm{m}$. However, there is at least one important problem involving IR radiation and droplets that violates this condition, namely, mist and cloud droplets that are of order $20 \mu \mathrm{m}$. Mist and cloud droplets are definitely spherical and cannot be modeled as Cartesian. Therefore, armed with the information that the advective affect on liquid temperature profile is negligible, we proceed to the droplet problem in spherical coordinates. The solution of the spherical droplet problem will then be applied to an experimental measurement of laser-induced droplet evaporation under both unsteady and QS conditions, and finally to the important problem of QS cloud droplet growth and evaporation under the influence of longwave infrared and shortwave solar radiation.

\section{Temperature Profile in a Spherical Droplet}


Next we consider the spherical or radial-coordinate version of the preceding problem (Fig. 1a): volumetric absorption of an inward pointing collimated radiant flux on a spherical droplet with conduction, neglecting advection, and initially neglecting emission by the droplet. The non-reflected part of the incident flux is $\mathrm{q}_{\mathrm{r}}$. Note a subtle change in the interpretation of $\mathrm{q}_{\mathrm{r}}$ (nonreflected incident flux) between this problem (finite sphere) and the semi-infinite slab. In the Cartesian (optically thick slab) problem the non-reflected flux $\left(q_{r}\right)$ was the same as the absorbed flux because no part of $q_{r}$ could escape the semiinfinite medium. Here part of $\mathrm{q}_{\mathrm{r}}$ might leave a finite droplet without having been absorbed. The fraction of $\mathrm{q}_{\mathrm{r}}$ absorbed by the droplet is quantified below using the absorption efficiency, $Q_{\mathrm{a}}$. The QS (no storage term) differential equation and boundary conditions are as follows:

$$
\begin{aligned}
& k_{\ell} \frac{d}{d r}\left(r^{2} \frac{d T}{d r}\right)-\left(1-f_{s}\right) q_{r} \frac{d}{d r}\left[r^{2}\left(e^{-k_{a}(R+r)}-e^{-k_{a}(R-r)}\right)\right]=0 ; \\
& \left.T\right|_{r=R}=T_{s} ;\left.\quad \frac{d T}{d r}\right|_{r=0}=0 .
\end{aligned}
$$

The differential equation is similar to the previous non-convective Cartesian problem except that, because the droplet is finite instead of semi-infinite, radiation can come from either side of the droplet to a point in the interior. The solution is

$$
\begin{aligned}
& \frac{T(r)-T_{s}}{T_{o}-T_{s}}=\frac{1+e^{-2 K_{a} R}-e^{-K_{a}(R-r)}-e^{-K_{a}(R+r)}}{\left(1-e^{-K_{a} R}\right)^{2}} ; \\
& T_{o}=\left.T\right|_{r=0}=T_{s}+\frac{\left(1-f_{s}\right) q_{r}}{k_{\ell} K_{a}}\left(1-e^{-K_{a} R}\right)^{2} ; \\
& q_{c, u}=q_{r} Q_{a, v} ; \quad Q_{a, v}=\left(1-f_{s}\right)\left(1-e^{-2 K_{a} R}\right),
\end{aligned}
$$

where $T_{0}$ is the center temperature and $Q_{a, v}$ is the volumetric absorption efficiency. An alternate way of expressing the temperature is in terms of the limiting temperature rise $\Delta \mathrm{T}_{\max }$ :

$$
\frac{T(r)-T_{s}}{\Delta T_{\text {max }}}=1+e^{-2 K_{a} R}-e^{-K_{a}(R-r)}-e^{-K_{a}(R+r)} ; \Delta T_{\max }=\frac{\left(1-f_{s}\right) q_{r}}{k_{\ell} K_{a}} ;
$$




$$
\frac{T_{0}-T_{s}}{\Delta T_{\max }}=\left(1-e^{-K_{a} R}\right)^{2} .
$$

The non-dimensional temperature relative to $\Delta \mathrm{T}_{\max }$ is plotted in Fig. $7 \mathrm{a}$ for $1 / \mathrm{K}_{\mathrm{a}}=$ $20 \mu \mathrm{m}$ (mid-IR) for a range of droplet radii from $\mathrm{R}=10$ to $200 \mu \mathrm{m}$. Due to the finiteness of a sphere, $\Delta T_{\text {max }}$ is not necessarily realized in the droplet (it would not be realized for optically thin droplets), as shown in Fig. 7a. For optically thick droplets, i.e., $R>>1 / K_{a} \sim 20 \mu \mathrm{m}$ (mid-IR), terms like $\exp \left(-K_{a}(R+r)\right)$ are negligible and $\Delta \mathrm{T}_{\max }$ is realized near the droplet center $\left(\left(\mathrm{T}_{0}-\mathrm{T}_{\mathrm{s}}\right) \rightarrow \Delta \mathrm{T}_{\max }\right)$. For optically thin droplets, i.e., $R<1 / K_{a} \sim 20 \mu \mathrm{m}$, nonlinear exponential terms become linear and the droplet center temperature rise $T_{0}-T_{S}$ approaches just a small fraction, i.e., $\left(K_{a} R\right)^{2}$, of $\Delta T_{\text {max }}$.

Next, in anticipation of the unsteady droplet problem in the next section, we consider whether or under what conditions the isothermal assumption is valid inside a droplet. This is similar to evaluating the Biot number when gas convection/conduction dominates heat transfer to the droplet with negligible phase-change energy. In the limit that gas convection becomes pure conduction $\left(\mathrm{Nu}_{R}=1\right)$, the Biot-number condition (with negligible radiation and latent heat) is: $\mathrm{Bi}=\mathrm{k} / \mathrm{k},<1$. This ratio is approximately equivalent to the temperaturedifference ratio $\left|T_{0}-T_{s}\right| /|\Delta T|$, where $\Delta T=T_{s}-T_{e}$ is the temperature change outside the droplet in the gas phase. Thus for conduction-dominated heating, the droplet is essentially isothermal because of the small ratio of thermal conductivities. When radiation dominates droplet heating, a radiative Biot number $\mathrm{Bi}_{\mathrm{r}}$ can be defined similarly as the product of two factors:

$$
B i_{r}=\frac{\left(T_{o}-T_{s}\right)}{\Delta T_{\max }} \cdot \frac{\Delta T_{\max }}{\Delta T}=\left(1-e^{-k_{a} R}\right)^{2} \cdot \beta_{r} ; \beta_{r}=\frac{\Delta T_{\text {max }}}{\Delta T}=\frac{\left(1-f_{s}\right) q_{r}}{k_{\ell} K_{a} \Delta T} .
$$

The first factor, as noted above, accounts for droplet optical thickness; it approaches unity for optically thick droplets. The second factor, the nondimensional parameter $\beta_{\mathrm{r}}$, has elsewhere [10] been observed to play the role of validating (when $\beta_{r}<1$ ) the surface absorption assumption in modeling transient radiative heating with in-depth absorption but without interfacial phase change in the Cartesian problem (semi-infinite solid). The same parameter appears here in the droplet problem playing a related role by validating the surface absorption/isothermal liquid assumptions (recall that these go together for optically thick conditions) when $\beta_{r} \ll 1$. Actually the condition $\beta_{r} \ll<1$ is sufficient but not necessary. Note that $\beta_{\mathrm{r}}$ has a singularity when $\Delta \mathrm{T} \rightarrow 0$, which did not arise in [10] because surface evaporation was not included. With surface evaporation included, it is certainly physically possible to have $\Delta T=0$, as considered above. This could easily cause $\beta_{r}>1$ and yet a droplet still be nearly isothermal. We can eliminate the singularity by removing phase-change so that 
the conductive heat flux is continuous across the interface (replace $\Delta \mathrm{T}$ with $\left.\mathrm{Rq}_{\mathrm{r}} / \mathrm{k}\right)$ :

$$
\left|q_{f g}\right| \ll\left|q_{c, s}\right|: B i_{r}=\frac{k\left(1-f_{s}\right)\left(1-e^{-k_{a} R}\right)^{2}}{k_{\ell} K_{a} R}=\left\{\begin{array}{l}
\frac{k\left(1-f_{s}\right) K_{a} R}{k_{\ell}}, K_{a} R \ll 1 \\
\frac{k\left(1-f_{s}\right)}{k_{\ell} K_{a} R}, K_{a} R \gg 1
\end{array}\right.
$$

This shows that with negligible phase change energy contribution: $\mathrm{Bi}_{\mathrm{r}}<<1$; the droplet is isothermal no matter the optical depth $\mathrm{K}_{\mathrm{a}} \mathrm{R}$.

When phase-change is not negligible, the magnitude of $\mathrm{Bi}_{\mathrm{r}}$ is not a direct indicator of the isothermal assumption; however, $\left(T_{0}-T_{s}\right)$ can be evaluated directly using Eq. (22b) for various fluxes and droplet sizes. The results for $\left(T_{0}-\right.$ $T_{s}$ ) for a relatively large droplet $R=100 \mu \mathrm{m}$ and relatively high flux $q_{r}=5000$ $\mathrm{W} / \mathrm{m}^{2}$ are shown in Fig. $7 \mathrm{~b}$. Over the spectral range $(0.2-200 \mu \mathrm{m})$ the maximum value of $\left(T_{0}-T_{s}\right)$ is about $0.3 \mathrm{~K}$, which occurs at 2, 2.6, and $3.6 \mu \mathrm{m}$. An error of $0.3 \mathrm{~K}$ in $T_{s}$ in evaluating quantities such as $P_{s}, h_{h g}$ or gas-phase fluxes would be inconsequential to droplet evaporation dynamics. Thus, liquid droplet radiative analysis supports treating droplets as spatially isothermal for QS evaporation.

Although this radiative formulation is for a particular kind of directionalspatial flux field-spherically isotropic, collimated flux - the solution can be applied or extended to other isotropic or quasi-isotropic radiation fields. For isotropic, diffuse irradiation, an exact analytic integral over the varying pathlengths in the spherical geometry is possible (if refraction at the interface is neglected). The solution has the simple Beer's law exponential transmissivity term (denoted by $\tau_{v}$ for volumetric transmissivity) in the absorption efficiency replaced with a slightly more complex expression,

$$
\mathrm{Q}_{\mathrm{a}, \mathrm{v}}=\left(1-\mathrm{f}_{\mathrm{s}}\right)[1-\underbrace{\mathrm{e}_{\tau_{\mathrm{v}}}^{-\mathrm{t}}}_{\text {isotropic-collimated }}] \rightarrow \mathrm{Q}_{\mathrm{a}, \mathrm{v}}=\left(1-\mathrm{f}_{\mathrm{s}}\right)[1-\underbrace{\frac{2}{\mathrm{t}^{2}}\left(1-(1+\mathrm{t}) \mathrm{e}^{-\mathrm{t}}\right)}_{\text {isotropic-diffuse or unidirectional-collimated irradiation }}] ; \mathrm{t}=2 \mathrm{~K}_{\mathrm{a}} \mathrm{R}
$$

where $t$ is optical depth along the sphere diameter. Note that the absorption efficiency on the right-hand side (RHS) of the arrow (for isotropic, diffuse irradiation) is the same as in Anomalous Diffraction theory for unidirectional, collimated irradiation. That is, fixing the radiation direction as being incident from one side of the droplet and varying the location of incidence over the surface of the droplet (and thus the pathlength through the droplet), again neglecting refraction and reflection at the interface, results in the same integral over pathlength. Therefore the expression on the RHS of the arrow above can be used for volumetric radiative properties (e.g., absorption coefficient) in a radiative transfer equation description of spherical droplet assemblies. It can be used as 
is, if neglect of interface refraction and reflection is tolerable. For the actual optical properties of water in air, correction for refraction and reflection at the droplet interface (which have been neglected throughout this analysis) is feasible and would be advisable. Interface absorption (the $\mathrm{f}_{\mathrm{s}}$ term) has been included.

Thus far emission by the droplet has been neglected. With the isothermal droplet assumption made, it is a simple matter to put emission into the problem by subtracting the blackbody hemispherical flux based on droplet temperature from the non-reflected, incident hemispherical flux: i.e., take $\mathrm{q}_{r}$ as $\mathrm{q}_{\mathrm{r}}$ (incident) $\mathrm{e}_{b}\left(T_{s}\right)$, (assuming temporarily that droplet emission and absorption efficiencies are equal and given by $Q_{a}$ ); henceforth $q_{r}$ will stand for this, the actinic, flux. The droplet temperature $T_{s}$ is assumed spatially isothermal by the reasoning above. When droplets are subject to strong SW incident flux (e.g., solar or SW laser flux) the assumption of equal droplet absorption and emission efficiencies will need to be changed to reflect the much smaller absorption efficiency of SW radiation compared with the often near-unity emission efficiency at LW. When droplets are not subject to strong collimated flux (e.g., solar or laser flux) and the incident hemispherical flux can be represented by a simple blackbody hemispherical flux, it is convenient to introduce the concept of radiative heat transfer coefficient. For a radiative environment that behaves as a blackbody at $T_{r}$ surrounding the entire droplet, the net radiative flux into the droplet can be written as:

$$
Q_{a} q_{r}=Q_{a} \sigma\left(T_{r}^{4}-T_{s}^{4}\right)=h_{r}\left(T_{r}-T_{s}\right) ; h_{r}=Q_{a} \sigma\left(T_{r}^{2}+T_{s}^{2}\right)\left(T_{r}+T_{s}\right) .
$$

If only part of the space around the droplet is at a different radiative temperature, e.g., for a droplet at the top of a cloud, suitable modifications can be made to $h_{r}$, as described below.

\section{Isothermal Unsteady Droplet Equations}

For the vapor-phase part of the droplet problem, the basic, heat/mass transfer theory results are the same as above (Cartersian) with the vapor length scale $L$ replaced by droplet radius $R$ :

Gas-side conductive heat flux: $q_{c, s}=\overbrace{N u_{R}}^{1} \frac{k}{R}\left(T_{s}-T_{e}\right)$;

Mass-transfer rate: $\dot{m}^{\prime \prime}=\frac{\rho \alpha_{m}}{R} \ln \left(1+B_{m}\right)$.

Introducing the unsteady droplet mass and energy balance equations, with the latter modified to account for the possibility of radiation coming to the interface from both sides as with non-opaque droplets, gives: 
Droplet mass balance: $\rho_{\ell} \frac{\mathrm{dR}}{\mathrm{dt}}=-\dot{\mathrm{m}}^{\prime \prime}$

Droplet energy balance (s-surface):

$$
\rho_{\ell} C \frac{R}{3} \frac{d T_{s}}{d t}=-q_{c, s}+q_{r} Q_{a}-\dot{m}^{\prime \prime} h_{f g} ; Q_{a}=1-\left(1-f_{s}\right)^{2} e^{-K_{a} 2 R}
$$

Droplet energy balance (u-surface):

$$
\rho_{\ell} C \frac{R}{3} \frac{d T_{s}}{d t}=-q_{c, u}+q_{r} Q_{a, v} ; Q_{a, v}=\left(1-f_{s}\right)\left[1-e^{-K_{a} 2 R}\right]
$$

Interface energy balance:

$$
\mathrm{q}_{\mathrm{c}, \mathrm{u}}+\mathrm{q}_{\mathrm{r}} \mathrm{Q}_{\mathrm{a}, \mathrm{s}}=\mathrm{q}_{\mathrm{c}, \mathrm{s}}+\dot{\mathrm{m}}^{\prime \prime} \mathrm{h}_{\mathrm{fg}} ; \mathrm{Q}_{\mathrm{a}, \mathrm{s}}=\mathrm{f}_{\mathrm{s}}\left[1+\left(1-\mathrm{f}_{\mathrm{s}}\right) \mathrm{e}^{-\mathrm{K}_{\mathrm{a}} 2 \mathrm{R}}\right]
$$

The latter two equations are not necessary for solving the problem (i.e., they are not independent) but are included to highlight the differences with the optically thick (semi-infinite liquid) Cartesian equations. In the s-surface equation, $Q_{a}$ represents the effective absorption efficiency of the droplet including both surface and volumetric absorption contributions. The single-surface contribution is $f_{\mathrm{s}}$, the effective two-surface contribution ( $>f_{s}$ for non-optically thick droplets) is $Q_{a, s}$, the volumetric contribution is $Q_{a, v}$, and the total is:

$$
Q_{\mathrm{a}}=\mathrm{Q}_{\mathrm{a}, \mathrm{v}}+\mathrm{Q}_{\mathrm{a}, \mathrm{s}}
$$

In the expressions for absorption efficiency in Eqns. (29-31), the Beers law transmissivity term compatible with the isotropic-collimated case (see Eqn. (24)) has been retained. The interface equation, Eqn. (31), replaces Eqn. (1) from the Cartesian problem.

For large, volumetrically optically thick droplets ( $t>>1$ ), the surface absorption efficiency is the single-interface value: $Q_{a, s} \sim f_{s}$, while the volumetric absorption efficiency is the remainder: $Q_{a, v} \sim 1-f_{s}$. For droplets that are optically thin both volumetrically and interfacially $\left(t<<1, Q_{a, v}<<1, f_{s}<<1\right)$, the surface absorption efficiency is effectively, nearly doubled from the single-interface value: $Q_{a, s} \sim 2 f_{s}$. The volumetric absorption efficiency is linear in $D: Q_{a, v} \sim t$, for the isotropic-collimated case, and $Q_{a, v} \sim 2 t / 3$, for the isotropic-diffuse/unidirectionalcollimated case. (The $2 / 3$ factor plays the role of converting droplet diameter $D$ to geometric mean beam length 2D/3.) Note that the surface absorption efficiency remains a constant with decreasing $D$ while the volumetric absorption efficiency vanishes. The existence of interfacial absorption (e.g., phase-change radiation) could play an important role in aerosol activation behavior in the small-D regime of fog and cloud microphysics where solute effects become important.

The characteristic time constant for the transient period depends on which terms in the energy balance dominate. In most situations the conduction term will be important whereas the radiation and phase-change terms may or may not be 
important, depending on conditions. Therefore, the time constant $\tau$ can be estimated by taking a balance between the storage and conduction terms. Approximating $d T_{s} / d t$ as $\left|T_{s}-T_{e}\right| / \tau$ and assuming quiescent gas conditions $\left(\mathrm{Nu}_{R}\right.$ =1) we obtain:

$$
\tau=\frac{\rho_{\ell} \mathrm{CR}^{2}}{3 \mathrm{k}}
$$

for the time constant. Note that although the thermophysical property ratio on the RHS has the appearance of inverse thermal diffusivity, it is not, as $k$ is for the gas while density and specific heat are for the liquid. (A similar but different mixed ratio appeared earlier in the definition of $\delta_{\mathrm{g}}$ with gas $\mathrm{k}$, liquid density, and gas specific-heat.) The time constant is a strong (quadratic) function of droplet size. Representative $\tau$ values for clouds, drizzle and rain, respectively, are as follows. For clouds, $R=10 \mu \mathrm{m}\left(\mathrm{Nu}_{\mathrm{R}}=1\right.$ would definitely be valid): $\tau=5 \mathrm{~ms}$; for drizzle, $\mathrm{R}$ $=100 \mu \mathrm{m}\left(\mathrm{Nu}_{\mathrm{R}}=1\right.$ should still be valid but approaching the upper size limit): $\tau=$ $0.5 \mathrm{~s}$; for rain, $\mathrm{R}=1000 \mu \mathrm{m}: \tau=50 \mathrm{~s}$. The last estimate (rain, $50 \mathrm{~s}$ ) is an upper limit because it is still based on $\mathrm{Nu}_{R}=1$; in reality the time constant would be smaller because convective effects would impose $\mathrm{Nu}_{R}>1$ (but in the spirit of being a time scale it is still indicative of the order of magnitude).

\section{Comparison with Large-Droplet $\mathrm{CO}_{2}$ Laser Experiment}

As a case to test the radiation-assisted evaporation theory including transient effects we select a reported experimental study wherein single, suspended 1-mm water droplets were heated and evaporated using a $\mathrm{CO}_{2}$ laser [11]. Droplet size was measured interferometrically using the diffracted laser light and confirmed photographically. In some tests where the droplet was suspended on a thermistor bead, droplet temperature was also measured. Typical droplet sizes ranged from 1 to $2 \mathrm{~mm}$. The location of the experiment was Salt Lake City, Utah so the relative humidity was probably fairly dry $(25 \%$ was selected based on reported evaporation rates without laser assistance) and the pressure (86 $\mathrm{kPa})$ was adjusted for altitude. The reported ambient temperature was $\mathrm{T}_{e}=22^{\circ} \mathrm{C}$.

The laser flux was $16,500 \mathrm{~W} / \mathrm{m}^{2}$. Since this flux is uni-directional it is appropriate to divide by 4 (the ratio of surface to cross-sectional area) in applying the model above, which is based on isotropic collimated flux, giving $4125 \mathrm{~W} / \mathrm{m}^{2}$. To account for laser energy absorbed by the support rod (which was blocked initially but became exposed as evaporation proceeded) the assumed constant value of $\mathrm{q}_{\mathrm{r}}$ in the calculation was increased (somewhat arbitrarily but by comparison with calculations) to $5500 \mathrm{~W} / \mathrm{m}^{2}$. Inverse absorption coefficient was taken as $20 \mu \mathrm{m}$, although aside from insuring that the droplet is optically thick, the precise value is unimportant. To account for non-quiescent air effects the following standard correlation [7] for natural convection was used. 


$$
\mathrm{Nu}_{\mathrm{R}}=1+0.215 \cdot \mathrm{Ra}_{\mathrm{D}}^{1 / 4} ; \quad \mathrm{Ra}_{\mathrm{D}}=\frac{\left(\mathrm{T}_{\mathrm{s}}-\mathrm{T}_{\mathrm{e}}\right) g \mathrm{D}^{3}}{\mathrm{Tv} \beta_{\mathrm{h}}}
$$

The particular test condition selected for theoretical comparison was a droplet with initial radius $\mathrm{R}=920 \mu \mathrm{m}$ at ambient temperature. The physical test was run by suspending a droplet and allowing it to cool by evaporative cooling (mostly conductive heat transfer) to near the wet-bulb temperature before exposing it to the laser flux. The calculation simulates the entire physical process. For the calculation, the unsteady mass and energy equations were integrated numerically. The results, experimental and theoretical, are shown in Fig. $8 a$ for $T_{s}(t)$ and Fig. $8 b$ for $R(t)$. The time scale is adjusted so that $t=0$ corresponds to the laser being turned on. Except for oscillations in the latter part of the test, most of the temperature history (Fig. 8a) is well represented by the theory, including both the initial cooling period $(t<0)$ and the laser-heating period $(t>0)$. Within each of these periods there is an initial transient response followed by $Q S$ behavior. The laser-off period $(t<0)$ shows that conduction-dominated $Q S$ evaporation is characterized by constant $T_{s}$ while the laser-on period $(t>0)$ shows that radiation-dominated QS evaporation is characterized by decreasing $\mathrm{T}_{\mathrm{s}}(\mathrm{t})$ for a droplet this large. The temperature oscillations for $\mathrm{t}>0$ are due to unsteady laser-induced circulation within the droplet. In Fig. $8 \mathrm{~b}$ the slope of the theoretical curve ( $\mathrm{dR} / \mathrm{dt})$ is not as steep as the experimental slope; however, that particular experiment was conducted with a thermistor (a relatively good thermal conductor) as support rod. Other experimental data obtained with non-conducting support rods showed consistently smaller slope magnitudes, i.e., $|\mathrm{dR} / \mathrm{dt}|$ values more consistent with the calculated curve. In all, the theory seems adequate for the radiation-assisted droplet evaporation situation, even when transient effects are important. Of course, it is a much easier problem if unsteadiness can be ignored and quasi-steady liquid assumed. Many important situations fall in this category, including clouds, which occupy the remainder of the discussion.

\section{Quasi-Steady Droplet Equations}

For process times much greater than the characteristic transient time ( $t>>$ $\tau)$, the QS approximation is valid. This is a significant mathematical simplification because the droplet energy equation changes from differential to algebraic. The QS assumption involves neglecting the LHS of the droplet energy equation to give, for the s-surface equation,

$$
0=-q_{c, s}+q_{r} Q_{a}-\dot{m}^{\prime \prime} h_{f g}
$$

and for the u-surface equation,

$$
0=-q_{c, u}+q_{r}\left(1-f_{s}\right) Q_{a, v}
$$


It can be shown that the s-surface equation above is equivalent to the equations presented earlier in terms of $B_{m}$ and $B_{h}$ for the opaque slab problem (which also apply to an opaque droplet by changing $L$ to $R$ ). If (a) the equations for mass flux involving $B_{m}$ and $B_{h}$ (which assume $Q S$ liquid) are combined, (b) adiabatic liquid is imposed, (c) vapor enthalpies are evaluated using the dilute (in water vapor) approximation, and (d) the liquid transmissivity factor $\left(\exp \left(-2 \mathrm{~K}_{\mathrm{a}} \mathrm{R}\right)\right)$ is appropriately accounted for (either included in the opaque $B_{m}-B_{h}$ equation or set to zero in the partially transmissive s-surface relations above), the same implicit, algebraic equation for QS droplet temperature is obtained. For dilute, QS conditions the droplet energy equation (s-surface) for $\mathrm{Nu}_{R}=1$ (no velocity slip) is:

$$
\Delta T \equiv T_{s}-T_{e}=\frac{R}{k}\left(q_{r} Q_{a}-\dot{m}^{\prime \prime} h_{f g}\right)
$$

where

$$
\dot{m}^{\prime \prime}=\frac{\rho \alpha_{m}}{R} \ln \left(1+B_{m}\right)=\frac{\rho \alpha_{m}}{R}\left(m_{1, s}-m_{1, e}\right) \quad \text { (dilute in water vapor). }
$$

This equation $(37,38)$ appears to be explicit in $T_{s}(L H S)$ but is actually implicit because of the RHS-terms $m_{1, s}\left(T_{s}\right)$ and $\operatorname{qr}_{r}\left(T_{s}\right)$. Still it is algebraic and can be solved for $T_{s}$ without integrating, which is the essential simplification of the QS droplet assumption. The water vapor mass fractions in (38) can be converted to partial pressures, $P_{1, s}$ and $P_{1, e}$, using the ideal gas assumption. The latter can be related to relative humidity $(\mathrm{RH})$ of the ambient environment (e-state). The former, assuming local thermodynamic equilibrium at the droplet surface, can be taken as the saturation pressure, $\mathrm{P}_{\mathrm{s}}\left(\mathrm{T}_{\mathrm{s}}\right)$, after adjustments for solute and curvature effects, as necessary.

It is common in the literature, particularly on cloud microphysics, to work with a combined droplet energy/mass equation. Combining the algebraic energy equation (37) with the ODE mass equation (28) gives:

$$
R \frac{d R}{d t}=\frac{k}{\rho_{\ell} h_{f g}}[\underbrace{T_{s}-T_{e}}_{\Delta T}-\frac{q_{r} Q_{a} R}{k}] .
$$

Now it can be seen from the QS equations what was observed above about the QS portions of the evaporating droplet $T_{s}(t)$ and $R(t)$ histories (Fig. 8). For conduction-dominated evaporation $\left(q_{r}=0\right), T_{s}$ is constant in the QS regime (Fig. $8 a$ ), therefore mass flux varies as $1 / R$, and $\mathrm{dR}^{2} / \mathrm{dt}$ is constant (the so-called $\mathrm{D}$ squared evaporation law).

Quasi-steady, conduction-driven evaporation $\left(\mathrm{q}_{\mathrm{r}}=0\right)$ : 


$$
\mathrm{T}_{\mathrm{s}}=\text { const } \dot{\mathrm{m}^{\prime \prime}}=\frac{\text { const }}{\mathrm{R}} ; \frac{\mathrm{dR}^{2}}{\mathrm{dt}}=\frac{-2 \mathrm{R} \dot{\mathrm{m}}^{\prime \prime}}{\rho_{\ell}}=\text { const } \text {. }
$$

In Fig. $8 b$ for $t<0$ the slope $d R / d t$ is actually not constant (which is barely discernible in the figure) but $\mathrm{dR}^{2} / \mathrm{dt}$ would be if $\mathrm{R}(\mathrm{t})$ were plotted as $\mathrm{R}^{2} \mathrm{vs}$. $t$. Thus the QS droplet $T_{s}(t)$ and $R(t)$ behavior for conduction-dominated evaporation (or condensation) is relatively simple mathematically. For radiation-dominated evaporation the situation is not quite as simple in the sense that nothing is really constant. However, evaluation at one unique state of radiation-dominated evaporation, namely, the condition when gas-phase conduction is zero $\left(q_{c, s}=0\right)$, gives some useful insight.

Quasi-steady, radiation-driven evaporation $\left(\mathrm{q}_{\mathrm{c}, \mathrm{s}}=0\right)$ :

$$
\left.\dot{\mathrm{m}}^{\prime \prime}=\mathrm{Q}_{\mathrm{a}} \mathrm{q}_{\mathrm{r}} / \mathrm{h}_{\mathrm{fg}}=\text { const (w.r.t } \mathrm{R}(\mathrm{t})\right) ; \frac{\mathrm{dR}}{\mathrm{dt}}=\frac{-\dot{\mathrm{m}}^{\prime \prime}}{\rho_{\ell}}=\text { const (w.r.t } \mathrm{R}(\mathrm{t}) \text { ). }
$$

It may appear from this that $\mathrm{dR} / \mathrm{dt}$ is constant in time since it is constant with respect to $R$; however, since $R$ varies in time, so do $\dot{m}^{\prime \prime}, d R / d t$, and $T_{s}$. In other words, $q_{c, s}$ can't stay zero unless $T_{e}$ changes in time with $T_{s}$; but we've already assumed $\mathrm{T}_{\mathrm{e}}$ to be constant. The QS portion of $\mathrm{T}_{\mathrm{s}}(\mathrm{t})$ in Fig. 8a for $\mathrm{t}>0$ shows this behavior with $\mathrm{T}_{\mathrm{s}}$ decreasing in time.

While the previous example for a water droplet of raindrop proportions showed regimes where transient effects were important over time scales of interest in relation to other physical phenomena, much smaller droplets such as clouds, have much smaller transient time scales and can often be approximated as QS. In the remainder of this discussion we consider the important problem of QS cloud-droplet phase-change with radiation effects.

\section{Cloud Droplets: Quasi-Steady Evaporation and Condensation near Saturation Conditions}

Until 1976 thermal radiation was largely neglected in theoretical descriptions of cloud microphysics other than in radiation fogs. Previous analyses [12] had shown that the magnitude of the temperature difference $\left(\Delta T=T_{s}-T_{e}\right)$ between cloud droplets $\left(T_{s}\right)$ and surrounding air $\left(T_{e}\right)$ was only of order $10^{-2} \mathrm{~K}$. Based on such a small number, it would be natural to think that thermal radiation was insignificant in cloud droplet growth. However, there are two problems with such thinking. The first is assuming the gas temperature to be the effective radiating temperature of the environment around the droplet. The second is failing to look at the contribution of thermal radiation relative to other heat transfer modes.

As early as 1877 Osborne Reynolds [13] realized that the effective environmental radiating temperature $\left(T_{r}\right)$ for droplets near the edge of clouds, particularly near the top, could be much lower than the cloud temperature, such 
that the magnitude of the temperature difference driving radiation could be much greater than $|\Delta T|$. Still, when realistic top-of-cloud sky temperatures are put into droplet energy-mass transfer analysis, as illustrated below, $|\Delta \mathrm{T}|$ induced by thermal radiation is only of order $10^{-2} \mathrm{~K}$ at most for realistic cloud droplets. Does this mean that radiation cannot be an important factor in droplet growth dynamics? No, and this is the second problem with misinterpreting the small magnitude of $\Delta \mathrm{T}$ : failing to look at the contribution of thermal radiation relative to other heat transfer modes. If $|\Delta T|$ induced by thermal radiation at cloud top is only of order $10^{-2} \mathrm{~K}$, that is still the same order as $|\Delta \mathrm{T}|$ induced by conduction or condensation. So Reynolds had made an important observation, and some in the decades after him referred to this as the Reynolds effect. Nevertheless, for many years Reynolds' effect was largely ignored.

During the same decades of the early 1900s in which the Reynolds effect was being largely overlooked, a significant mystery was puzzling the atmospheric science community: the problem of how micron-sized cloud droplets could become millimeter-sized raindrops by diffusion only, with negligible droplet inertia (velocity slip). Not all of this process was a mystery: in "cold" clouds, where part of the cloud contained ice particles, the differential vapor pressure between liquid and solid water could cause ice particles to grow at the expense of liquid droplets. This process, the so-called Bergeron (or Wegener-Bergeron-Findeisen) process, was elucidated in the early 1900s, and helped to explain how precipitation forms in mixed-phase clouds. But in clouds that are everywhere above the freezing point, so-called "warm" clouds, where it would not be possible to have ice crystals, rain had been observed to form from liquid cloud droplets in under an hour. The mechanism whereby this happens is still considered an unsolved problem in atmospheric science. This problem has been called the "condensation-coalescence bottleneck" because of the absence of a mechanism to explain liquid droplet growth between stable cloud size, roughly $20-\mu \mathrm{m}$ diameter, and the size where inertial effects, e.g., turbulence-induced coagulation, can take over, roughly $80-\mu \mathrm{m}$. Reynolds' hypothesis about radiationassisted droplet growth had been considered as a possible mechanism for raindrop growth in early years [14] but not in a comprehensive way, either experimentally or theoretically.

In 1976 Roach [15] included LW radiation in classical quasi-steady cloud droplet growth theory and showed that radiation could be important in the size range of stable cloud droplets, $20 \mu \mathrm{m}$. Still, he contended that ground fog was the main physical manifestation of radiation effects. Here we re-visit the warm cloud precipitation question by applying droplet radiation analysis to cloud droplets, similar to Roach, and we consider the effect of radiation on cloud droplet evolution and stability as well as the possible role of radiation in growing cloud droplets through the condensation-coalescence bottleneck.

Cloud droplets are typically assumed to be QS over time scales of importance fluid mechanically in cloud dynamics; they also exist in a nearly saturated thermodynamic environment. Therefore we specialize the QS droplet mass and energy equations developed above to conditions near saturation. First we introduce the variable called supersaturation, $s$, defined as relative humidity 
minus one: $s=R H-1$. Then we apply the concept of supersaturation to the thermodynamic e-state, which is never far from saturation in a cloud (perfect saturation over a flat interface of pure water means $s=0$ ). A typical magnitude of $s$ in stable clouds is less than a fraction of a percent. The largest values occur in developing convective clouds with strong vertical motions. In these clouds adiabatic cooling induced by updrafts with velocities of the order meters per second can drive s as high as a few percent, but only for a few minutes or tens of minutes. Then $s$ returns to a fraction of a percent as thermodynamic equilibrium is re-established (that $s$ does not go identically to zero is due primarily to the Kelvin effect, which is the surface-tension or droplet curvature effect). In the core of the cloud the sign of $s$ is generally positive; on the edges it can fluctuate positive or negative due to mixing with drier surrounding air. But in either case the magnitude is small such that near-saturation conditions prevail: $|\mathrm{s}|<<1$.

Next we introduce the Clausius-Clapeyron equation to describe the variation of saturation pressure (equilibrium vapor-pressure over a flat interface) with temperature. In integrated form (assuming $h_{\mathrm{fg}}$ constant, which is valid for small deviations from saturation) the Clausius-Clapeyron equation is:

$$
\frac{P_{s}\left(T_{s}\right)}{P_{s}\left(T_{e}\right)}=\exp \left[\frac{h_{f g}}{R}\left(\frac{1}{T_{e}}-\frac{1}{T_{s}}\right)\right] .
$$

Furthermore for small supersaturation values we can introduce the following approximation:

$$
\frac{\mathrm{h}_{\mathrm{fg}}|\Delta \mathrm{T}|}{\mathrm{RT}^{2}} \ll<1 ; \mathrm{T}_{\mathrm{s}} \approx \mathrm{T}_{\mathrm{e}}(=\mathrm{T}) ; \Delta \mathrm{T} \equiv \mathrm{T}_{\mathrm{s}}-\mathrm{T}_{\mathrm{e}} .
$$

Here $\Delta T$ is the (small) deviation of the droplet temperature from the environment (air) temperature. It is "small" because of, or, in the sense that, $|\mathrm{s}|<<1$. Now Taylor series expansions are applied to the quasi-steady mass and energy equations (s-surface) above, Eqs. (37)-(39), to obtain (see $[15,16]$ for details) the following approximate explicit relations for droplet temperature and size (switching from droplet radius $\mathrm{R}$ to diameter $\mathrm{D}$ ):

$$
\Delta \mathrm{T} \approx \frac{\mathrm{s}+\mathcal{Q}_{\mathrm{r}} \mathrm{D} \frac{\Psi}{\mathrm{H}}-\frac{\mathrm{D}_{\mathrm{C}}}{\mathrm{D}}}{\mathrm{H}+\Psi} ; \quad \frac{\mathrm{d} \mathrm{D}^{2}}{\mathrm{dt}} \approx \frac{\Gamma\left(\mathrm{s}-\mathcal{Q}_{\mathrm{r}} \mathrm{D}-\frac{\mathrm{D}_{\mathrm{C}}}{\mathrm{D}}\right)}{\mathrm{H}+\Psi}
$$

where: $\mathrm{H}=\frac{h_{\mathrm{fg}}}{R_{\mathrm{w}} T^{2}} ; \Psi=\frac{k R_{w} T}{\alpha_{m} h_{f g} P_{s, e}}=\frac{1.61 P C_{p}}{P_{s, e} h_{f g} L e}$; 


$$
\Gamma=\frac{8 \mathrm{k}}{\rho_{\ell} \mathrm{h}_{\mathrm{fg}}} ; \mathcal{Q}_{\mathrm{r}}=\frac{\mathrm{Q}_{\mathrm{a}} \mathrm{q}_{\mathrm{r}} \mathrm{H}}{2 \mathrm{k}} .
$$

The first relation above (for $\Delta \mathrm{T}$ ) is the QS algebraic droplet energy equation. The second relation (for $\mathrm{dD}^{2} / \mathrm{dt}$ ) is the combined (algebraic) energy and (differential) mass equations. The quantity $D_{c}$ is the effective diameter for the Kelvin effect, which is introduced when the equilibrium water vapor partial pressure at the droplet surface $\mathrm{P}_{1, \mathrm{~s}}$ is corrected for curvature (surface tension), and is typically of order $10^{-3} \mu \mathrm{m}$. The effective Kelvin diameter can be defined in terms of more basic physical properties such as surface tension and can be found in [16]. The Kelvin term, with a $\mathrm{D}^{-1}$ dependence, becomes less important for increasing $D$ values. The solute term, which has a $D^{-4}$ dependence, is not shown because it requires assumptions about the solute and it becomes less important at increasing diameters where radiation becomes important. The term $\mathrm{P}_{\mathrm{s}, \mathrm{e}}$ means $\mathrm{P}_{\mathrm{s}}\left(\mathrm{T}_{\mathrm{e}}\right)$. These approximate explicit relations appear in atmospheric science literature routinely (e.g., reference books [16-17]) without the radiation term (but including solution and curvature effect terms and are called Kohler theory or Kohler curves). The radiation term appears occasionally in research papers [e.g., 18-23], particularly those on fogs [18-20]. The first parameter usually appears as:

$$
H=\frac{1}{T}\left(\frac{h_{f g}}{R T}-1\right) ;(\text { alternative definition). }
$$

However, we find the former definition (Eq. (45), which is within the small supersaturation approximation) matches the more accurate, implicit equations better. We apply these results to droplets large enough that solution and curvature $\left(D_{c}\right.$ term) effects can be neglected, i.e., the water is pure and the saturation pressure at the s-surface is that over a flat surface.

The explicit, QS, near-saturation equations above, which are relevant to cloud and fog micro-physics, show how radiation can cause the opposite effect on droplet growth to that of supersaturation (i.e., conduction only). For example, considering droplets near cloud top, radiant heating (e.g., solar, $\mathrm{q}_{r}>0$ ) can cause droplet evaporation $\left(\mathrm{dD}^{2} / \mathrm{dt}<0\right)$ even when the cloud environment is slightly supersaturated $(s>0)$. On the other hand, radiant cooling (e.g., to colder upper levels, $\left.\mathrm{q}_{\mathrm{r}}<0\right)$ can cause droplet condensation $\left(\mathrm{dD}^{2} / \mathrm{dt}>0\right)$ even when the cloud edge is slightly subsaturated $(s<0)$. The key to the effect of infrared radiation (including solar NIR) on cloud droplet growth is the relative magnitude of the nondimensional radiation term, $Q_{r} \mathrm{D}$, compared with the magnitude of supersaturation, $\mathrm{s}$. The radiation term depends on availability of solar radiation to the cloud top and the effective radiative temperature of the higher atmospheric layers seen by the cloud top, which depends on solar zenith angle, (day- or nighttime) and the presence and altitude of higher (colder) cloud layers. These 
radiative effects, combined with turbulent fluid mechanics and mixing between cloud matter and clear, drier air at the edge of the cloud, determine the growth of cloud droplets. The importance of turbulence and mixing in clouds seems to be universally recognized in atmospheric science research; the importance of infrared radiation in clouds (as opposed to fogs) appears to be less well recognized but nonetheless is recognized [17-23].

\subsection{Sample Cloud Droplet Radiation Calculation}

To illustrate the potential effect of radiation on cloud droplets the nondimensional radiative parameter $\mathcal{Q}_{\mathrm{r}} \mathrm{D}$ in the preceding relations for QS droplet temperature and growth/evaporation rate is evaluated for conditions that might be representative of lower troposphere, top-of-cloud, tropical to mid-latitude summertime with optically thick cooler upper level cloud layer present. The follow properties for air (dilute in water) at $\mathrm{T}_{\mathrm{e}}=20^{\circ} \mathrm{C}$ and $\mathrm{P}=70 \mathrm{kPa}$ are used:

$$
\begin{aligned}
& \rho=0.823 \frac{\mathrm{kg}}{\mathrm{m}^{3}} ; C_{\mathrm{p}}=1.0 \frac{\mathrm{kJ}}{\mathrm{kgK}} ; \mathrm{k}=2.60 \mathrm{e}-2 \frac{\mathrm{W}}{\mathrm{m} \cdot \mathrm{K}} ; v=2.16 \mathrm{e}-5 \frac{\mathrm{m}^{2}}{\mathrm{~s}} ; \\
& \alpha_{\mathrm{m}}=3.58 \mathrm{e}-5 \frac{\mathrm{m}^{2}}{\mathrm{~s}} ; \alpha_{\mathrm{h}}=3.11 \mathrm{e}-5 \frac{\mathrm{m}^{2}}{\mathrm{~s}} \\
& \mathrm{Pr}=0.695 ; \mathrm{Sc}=0.603 ; \mathrm{Le}=1.15 ; \\
& \mathrm{h}_{\mathrm{fg}}=2454 \frac{\mathrm{kJ}}{\mathrm{kg}} ; \mathrm{P}_{\mathrm{s}, \mathrm{e}}=2.315 \mathrm{kPa} .
\end{aligned}
$$

These properties result in the following intermediate parameters:

$\mathrm{H}=0.0619 \mathrm{~K}^{-1} ; \Gamma=84.76 \frac{\mu \mathrm{m}^{2}}{\mathrm{~s} \cdot \mathrm{K}} ; \Psi=0.0173 \mathrm{~K}^{-1}$.

For $\mathrm{D}=20 \mu \mathrm{m}$ and using Roach's [15] recommended average infrared absorption coefficient of $1 / \mathrm{K}_{\mathrm{a}}=4.3 \mu \mathrm{m}$, the absorption efficiency (which is both volumetric and total since surface absorption is assumed zero) is $Q_{a}=0.91$. For a droplet near the cloud top but with negligible solar radiation the half-space above it will approach blackbody behavior at an effective radiative temperature of the higher cloud layer $\mathrm{T}_{\mathrm{r}}$ while the half-space below will be black (for an optically thick cloud) at approximately the same temperature $T_{s}$; the net flux absorbed by the droplet will be: 


$$
\begin{aligned}
& Q_{a} q_{r}=Q_{a} \sigma(\underbrace{F_{s s}}_{1-F_{s r}} T_{s}^{4}+F_{s r} T_{r}^{4}-T_{s}^{4})=Q_{a} \sigma F_{s r}\left(T_{r}^{4}-T_{s}^{4}\right)=h_{r}\left(T_{r}-T_{s}\right) ; \\
& h_{r}=Q_{a} \sigma F_{s r}\left(T_{r}^{2}+T_{s}^{2}\right)\left(T_{r}+T_{s}\right) .
\end{aligned}
$$

For $\mathrm{T}_{\mathrm{r}}=0^{\circ} \mathrm{C}$, and view factor $\mathrm{F}_{\mathrm{sr}}=0.5$ (i.e., droplet at the top of a cloud with an ideal planar boundary),

$$
\mathrm{h}_{\mathrm{r}}=2.34 \frac{\mathrm{W}}{\mathrm{m}^{2} \mathrm{~K}} ; \mathrm{Q}_{\mathrm{a}} \mathrm{q}_{\mathrm{r}}=\mathrm{h}_{\mathrm{r}}\left(\mathrm{T}_{\mathrm{r}}-\mathrm{T}_{\mathrm{s}}\right)=-47 \frac{\mathrm{W}}{\mathrm{m}^{2}} ; Q_{\mathrm{r}}=-5.5 \mathrm{e}-5 \mu \mathrm{m}^{-1}
$$

This flux $\left(-47 \mathrm{~W} / \mathrm{m}^{2}\right)$ represents a spatial average over the droplet surface, and is obviously sensitive to assumed properties and temperatures but is still comparable to values estimated by Roach [15]. The corresponding nondimensional radiation parameter is $Q_{T} \mathrm{D}=-0.0011$, which means that for these conditions radiation has the same importance for droplet growth as conduction does with a super-or sub-saturation magnitude of $0.1 \%$. The droplet temperature and growth rate for a supersaturation of $s=0.001$ with (and without) radiation are:

$$
\Delta \mathrm{T}=0.00878^{\circ} \mathrm{C}\left(0.0126^{\circ} \mathrm{C}\right) ; \frac{\mathrm{dD^{2 }}}{\mathrm{dt}}=2.25 \mu \mathrm{m}^{2} / \mathrm{s}\left(1.07 \mu \mathrm{m}^{2} / \mathrm{s}\right) .
$$

This sample calculation illustrates that infrared cooling has a significant effect on the condensation growth rate of droplets near cloud top, with the growth rate for the illustrated conditions being doubled by radiation compared with that if only conduction were operative. This effect on droplet growth rate may seem somewhat surprising, given how small the temperature difference is between the droplet and ambient gas. As noted above, this smallness of $\Delta T$ led many early researchers to neglect radiative effects outright in the beginning of their analyses and thereby overlook this important effect [12].

\subsection{Integrated Droplet Size History for Constant Environment:}

The quasi-steady droplet growth equation above, neglecting curvature and solute effects, can be arranged as:

$$
\frac{\mathrm{dD}}{\left(\frac{\mathrm{s}}{\mathrm{D}}-\mathcal{Q}_{\mathrm{r}}\right)}=\frac{\Gamma / 2}{\mathrm{H}+\Psi} \mathrm{dt}
$$


If constant properties are assumed this equation is analytically integrable. Constant properties means constant environmental variables $\left(\mathrm{s}, \mathrm{T}=\mathrm{T}_{\mathrm{e}}, \mathrm{P}\right.$, and $\mathrm{q}_{\mathrm{r}}$ ) and therefore constant $\mathrm{H}(\mathrm{T}), \Psi(\mathrm{T}, \mathrm{P}), \Gamma(\mathrm{T})$, and $\mathcal{Q}_{\mathrm{r}}\left(\mathrm{T}, \mathrm{q}_{\mathrm{r}}\right)$. We note that this assumption neglects the droplet diameter dependence of volumetric absorption efficiency in the radiation parameter, which effect becomes important at smaller diameters $(D<10 \mu \mathrm{m})$; however, we are interested here in the effect of radiation at larger diameters. The general integral is:

$$
\text { General solution: } \frac{-\mathcal{Q}_{\mathrm{r}}\left(\mathrm{D}-\mathrm{D}_{\mathrm{o}}\right)-\mathrm{s} \cdot \ln \frac{\left|\mathrm{s}-\mathcal{Q}_{\mathrm{r}} \mathrm{D}\right|}{\left|\mathrm{s}-\mathcal{Q}_{\mathrm{r}} \mathrm{D}_{\mathrm{o}}\right|}}{\mathcal{Q}_{\mathrm{r}}^{2}}=\frac{\Gamma / 2}{\mathrm{H}+\Psi} \cdot \mathrm{t} .
$$

If radiation is neglected the conduction solution is:

$$
\text { Conduction solution: } \frac{\mathrm{dD}^{2}}{\mathrm{dt}} \approx \frac{\Gamma \mathrm{s}}{\mathrm{H}+\Psi} ; \mathrm{D}^{2}-\mathrm{D}_{\mathrm{o}}^{2}=\frac{\Gamma}{\mathrm{H}+\Psi} \mathrm{s} \cdot \mathrm{t} \text { (parabolic) }
$$

If conduction is negligible compared to radiation the integral is:

$$
\text { Radiation solution: } \frac{\mathrm{dD}^{2}}{\mathrm{dt}} \approx \frac{-\Gamma \mathcal{Q}_{\mathrm{r}} \mathrm{D}}{\mathrm{H}+\Psi} ; \mathrm{D}-\mathrm{D}_{\mathrm{o}}=\frac{-\Gamma / 2}{\mathrm{H}+\Psi} \mathcal{Q}_{\mathrm{r}} \cdot \mathrm{t} \quad \text { (linear) }
$$

Some sample results are plotted for droplet size history in Figs. 9 and 10 for a condition of radiant heat loss from the droplet to the cold upper level cloud layer, $\mathcal{Q}_{\mathrm{T}}=-5.5 \mathrm{e}-5 \mu \mathrm{m}^{-1}$. This is the same radiant loss condition as for the sample calculation above. Figure 9 shows the droplet size history when the rest of the parameters are also the same as the sample calculation above with an initial droplet size of $D_{0}=20 \mu \mathrm{m}$. For this case the supersaturation is positive at $\mathrm{s}$ $=0.001$, which means that in the absence of radiation heat transfer, the droplet would naturally experience growth by condensation. This history is the curve labeled "conduction" in Fig. 9. Above the "conduction" curve is the curve for radiation only (no conduction) labeled "radiation". Above both of those is the general solution for droplet size history when both radiation and conduction are included. This set of curves shows how, as noted above, the initial growth rate (slope) is doubled by radiation. Furthermore, because of the linear versus parabolic growth laws for radiation versus conduction, it shows that the effect of radiation is to reduce the time required for the droplet to grow from 20 to $40 \mu \mathrm{m}$ by more than a factor of two, from $1100 \mathrm{~s}$ to $420 \mathrm{~s}$ (almost a factor of three).

In Fig. 10 the supersaturation is changed from positive to negative, i.e., subsaturation with $s=-0.001$, which, in the absence of radiation, corresponds to droplet evaporation. That curve is labeled "conduction" and shows the droplet completely evaporating in $374 \mathrm{~s}$. The "radiation" only curve is, of course, the 
same as in Fig. 9, showing droplet growth by condensation, since it is not a function of $\mathrm{s}$. The combined-effect curve shows that the effect of radiation heat loss to the cold upper cloud layer is strong enough to change the droplet size history from evaporation to condensation. The growth rate is obviously not as fast in Fig. 10, reaching only to $26-\mu \mathrm{m}$ diameter after $1100 \mathrm{~s}$, compared with $114 \mathrm{~s}$ in Fig. 9 to reach $26 \mu \mathrm{m}$, but that is due to the opposite effect of conductionsupersaturation between the two figures; radiation has to work against conduction in Fig. 10 whereas conduction and radiation work together in Fig. 9 to support condensation growth.

One of the most important results of this model including radiation, shown in Fig. 9, is the implication for the warm rain or condensation-coalescence bottleneck problems. It has been known for a long time that since droplet growth

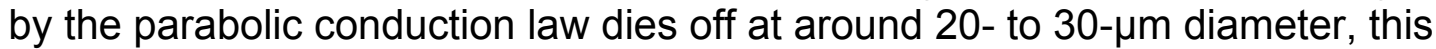
mechanism (diffusion/conduction without radiation) cannot explain the observed growth of cloud drops to precipitation (drizzle or rain) sized droplets. It cannot even explain the growth needed to achieve the size range where droplet coalescence induced by air turbulence and droplet inertia (slip) can take over, which is around $80 \mu \mathrm{m}$. However, as Fig. 9 shows, the linear radiation mechanism picks up at around $20 \mu \mathrm{m}$ and is able to grow droplets from 20 to 40 $\mu \mathrm{m}$ in times of the order of minutes, or to $80 \mu \mathrm{m}$ in several tens of minutes. At the 40- to $80-\mu \mathrm{m}$ size range, turbulence- and gravitational settling-induced droplet collision can begin to take over and grow droplets to precipitation sizes.

These calculations all assume that ambient supersaturation is constant. Of course condensational droplet growth takes water vapor from the ambient air. It is possible that evaporation of smaller droplets can make up some or all of the required water vapor. The balance of water would determine the variation in local and cloud-average supersaturation. This variation would have to be accounted for to determine the rate of growth of the largest droplets in clouds with a finite amount of water and a finite amount of space around each droplet. Nevertheless, these constant-supersaturation estimates provide an indication that the radiative mechanism is likely an important one in connecting the condensationcoalescence bottleneck for droplet growth in warm rain clouds.

Just as Figs. 9 and 10 demonstrate the effect of LW radiant emission in enhancing cloud droplet growth, the opposite effect of SW (solar) radiant absorption could be shown by including it [24-26] in the radiation term $\mathcal{Q}_{1} \mathrm{D}$, with the possibility that $S W$ radiation could change the sign from negative to positive. This would require estimating absorption of SW radiation, which is more complicated just by the stronger spectral variation of water's absorption coefficient in the SW range compared to the LW range and the smaller absorption efficiency for SW radiation compared with the near unity emission efficiency for LW radiation (different absorption and emission efficiencies would need to be evaluated, as noted previously). Still it is clear that SW absorption would tend to offset the effect of LW emission. For strong enough SW absorption the net radiation term could be positive, which would enhance droplet evaporation rate under sub-saturated $(s<0)$ conditions and possibly turn 
condensation under supersaturated $(s>0)$ conditions into evaporation, if the radiation term was big enough: $Q_{\mathrm{r}} \mathrm{D}>\mathrm{s}$.

Although the actual contributions of LW and SW depend strongly on atmospheric conditions, in almost all cases the contribution of LW near cloud top would be mostly one of emission, which is still very efficient for small cloud droplets, just as for the ocean. In contrast, the contribution of SW radiation would be one of absorption, and, unlike with ocean water, would not be very efficient with clouds because of the small droplet size relative to SW mean free photon path length. Taking into account these variable and offsetting effects it may be said in general terms that $Q_{a} q_{r}$ for top-of-cloud droplets is usually negative (net emission) by about -20 to $-30 \mathrm{~W} / \mathrm{m}^{2}$ even with the sun on cloud tops because of inefficient SW absorption and because of low effective radiative temperature of the atmosphere and space above. Still, the precise value of $Q_{a} q_{r}$ and thus $\mathcal{Q}_{r} \mathrm{D}$ depends strongly on sun position and higher clouds, with average $Q_{\mathrm{r}} \mathrm{D}$ values being of the same order of magnitude as the supersaturation in clouds. This means that radiation may be an important factor in determining cloud behavior on the time scale of an hour or more, although not during the initial rapid formation phase of convective, cumulus clouds, which occurs over minutes and is dominated by fluid dynamics and conductive/convective transfer. Whether radiation-augmented diffusive transport would actually be able to grow a cloud droplet from 20- to 40- $\mu \mathrm{m}$ diameter in an hour would also depend on the residence time of the droplet near the radiating boundary.

Another significant effect of radiation on cloud droplet size can be seen if we include the Kelvin effect term, set the droplet growth rate $\mathrm{dD} / \mathrm{dt}$ to zero, and solve for the equilibrium supersaturation as a function of droplet diameter for given $\mathrm{D}_{\mathrm{c}}$ and $\mathcal{Q}_{\mathrm{r}}$ :

$$
\mathrm{s}_{\mathrm{eq}}=\mathcal{Q}_{\mathrm{r}} \mathrm{D}+\frac{\mathrm{D}_{\mathrm{c}}}{\mathrm{D}} .
$$

Plots of $S_{\text {eq }}$ vs. D based on (61) represent the equilibrium balance between Kelvin and radiation effects; we will refer to these curves as Kohler curves, after the more common solute-Kelvin balance curves, which include the solute effect. A plot of (61) is shown in Fig. 11 with $\mathrm{D}_{\mathrm{c}}=2.4 \mathrm{e}-3 \mu \mathrm{m}$ and $\mathcal{Q}_{\text {, ranging from } 0 \text { to }}$ $\left( \pm 4.8 \mathrm{e}-5 \mu \mathrm{m}^{-1}\right) \mathrm{Q}_{\mathrm{a}}(\mathrm{D})$, corresponding to $\mathrm{q}_{\mathrm{r}}$ ranging from 0 to $\pm 40 \mathrm{~W} / \mathrm{m}^{2}$. Sizedependent absorption $Q_{a}(D)$ is estimated from Eq. (24) (unidirectional-collimated) with $\mathrm{f}_{\mathrm{s}}=0$ and $1 / \mathrm{K}_{\mathrm{a}}=4.3 \mu \mathrm{m}$. The $\mathrm{q}_{\mathrm{r}}=0$ broken-line curve is the Kelvin term.

The region above a curve is for $\mathrm{dD} / \mathrm{dt}>0\left(\mathrm{~s}>\mathrm{s}_{\mathrm{eq}}\right)$. A droplet at these conditions grows (moves to the right) by condensation. The region below a curve is for $\mathrm{dD} / \mathrm{dt}<0\left(\mathrm{~s}<\mathrm{s}_{\mathrm{eq}}\right)$. A droplet at these conditions shrinks (moves to the left) by evaporation. If we assume an infinite ambient (a reservoir of mass and energy), such that evaporation and condensation don't affect the ambient water vapor pressure or temperature $(s=$ constant), the state point moves horizontally at constant s. 
From these considerations it can be seen that $\mathrm{q}_{\mathrm{r}}<0$ curves represent unstable equilibrium because slope is negative everywhere. Any point starting to the left of the curve would move (at constant s) to the left, away from the curve. The droplet would evaporate until it encountered the solute branch of the Kohler curve (not shown). Any point starting on the right of the curve would move (at constant s) to the right, again, away from the curve. If s were constant, a droplet starting with an initial condition to the right of $a q_{r}<0$ curve (e.g., $D=20 \mu \mathrm{m}$ for $q_{r}$ $=-10 \mathrm{~W} / \mathrm{m}^{2}$ and $\mathrm{s}=0$ ) would grow indefinitely.

Since the ambient environment around an individual cloud droplet is not an infinite reservoir of mass and energy, droplet evaporation and condensation do affect the surrounding value of supersaturation. This, in effect, means that when an individual droplet state point moves to the left or right on a Kohler diagram by evaporation or condensation, it does so on a sloped curve with a negative slope determined by its own quasi-steady droplet mass/energy balance equations (44) and the mass and energy balances of the surrounding vapor, which are affected by the mass/energy balances of other nearby surrounding droplets. For example, if a given droplet and all of its nearby neighbors are evaporating, this evaporation puts water vapor into the droplet's local vapor surroundings and takes energy therefrom at nearly constant total pressure; therefore $s$ increases. As the size of that droplet $D$ (as well as the neighboring droplets) decreases (by evaporation), the superstation of the vapor in that neighborhood s increases; a state point initially to the left of the curve would move left and upward. Conversely, a local, net condensation effect would take water vapor from a given droplet's local surroundings and put energy into the surroundings; therefore s would decrease. As D increased (by condensation), s would decrease; a state point initially to the right of the curve would move right and downward. This would allow the change-of-state of an individual droplet in a finite environment to align itself with a portion of the Kohler curve-even a portion of the curve with a negative slope-and become stable. This stabilizing effect of a finite ambient has been analyzed and shown to be applicable to cloud conditions by Defour and Defay [27]; it explains why clouds can be stable when the Kelvin effect operating in an infinite ambient (i.e., a reservoir with $s=$ constant) would dictate that droplets above the $\mathrm{q}_{\mathrm{r}}=0$ curve (or above any $\mathrm{q}_{\mathrm{r}}<0$ curve) in Fig. 14 should grow by condensation indefinitely. Implications of the stabilizing effect of radiation can be further anticipated as follows.

Consider a net positive (gain of) radiation case, i.e., SW+LW absorption slightly stronger than LW emission. For example, Fig. 11 shows a curve for $\mathrm{q}_{\mathrm{r}}=5$ $\mathrm{W} / \mathrm{m}^{2}$ that exhibits a minimum at about $\mathrm{D}=20 \mu \mathrm{m}$ and $\mathrm{s}=0.0002$; for $\mathrm{D}>20 \mu \mathrm{m}$ the slope is positive. This positive-slope branch of the curve is therefore stable even in an infinite ambient environment. That is, a droplet with initial state above the curve $(\mathrm{dD} / \mathrm{dt}>0)$, would grow by condensation and move to the right (horizontally for $s=$ constant) until it encountered the positive-slope branch, and there it would be at stable equilibrium $\left(\mathrm{dD} / \mathrm{dt}=0, \mathrm{~s}=\mathrm{s}_{\mathrm{eq}}\right)$. The effect of net radiation gain, $q_{r}>0$, is to modify the $q_{r}=0$ Kohler curve in a way that droplets above the curve do not need to grow so large to become stable as they would have to for $q_{r}=0$ or $q_{r}<0$. 
Of course the preceding argument only applies to droplets above the curve; those below the curve move to the left by evaporation until they encounter the positive-slope solute branch (not shown) or the positive-slope radiation branch. The effective range of average cloud supersaturation for stable droplets for the conditions of Fig. $11\left(q_{r}>0\right)$ would be well within the range of typical supersaturations for stable clouds (accurate numerical predictions would require the solute term to be added). As $\mathrm{q}_{\mathrm{r}}$ increases the Kohler curve shifts upward and the minimum point moves to the left. Thus, higher values of positive $q_{r}$ tend to stabilize clouds with higher average supersaturations and smaller droplets. Lower values of positive $q_{r}$ or even negative $q_{r}$ values tend to stabilize clouds with lower average supersaturations, possibly sub-saturations, and larger droplets. The effect of radiation on cloud droplet stability, as evidenced by its effect on the radiation-Kelvin Kohler curve in the range of typical cloud droplet sizes, as shown in Fig. 11, could be quite significant.

\section{Summary}

The role of thermal radiation in water droplet evaporation and condensation has been investigated theoretically. The effects of distributed (volumetric) absorption versus conduction and convection (advection) have been studied. For realistic conditions it is found that advection within the liquid phase induced by surface regression can be neglected and conduction usually establishes a spatially isothermal droplet. Comparison with experimental results for a large, laser-irradiated droplet suggests that these assumptions are reasonable for predicting evaporation rate, even when the combination of droplet size and optical properties is such that internal radiation absorption is not volumetrically uniform, such as with collimated laser radiation (provided the laser flux is considered to be distributed over the droplet surface). Moreover, it is shown that absorption and emission can be treated as surface phenomena; indepth effects can be neglected. Extending the analysis to cloud droplets shows that ambient radiation, such as to cold upper levels at cloud top or with warm ground at cloud bottom, can have a significant effect on droplet growth behavior. For typical ambient conditions the thermal radiation effect near the edge, e.g., top, of a cloud can be as strong as the effect of a relatively small supersaturation, i.e., a magnitude of $0.1 \%$. This could be enough to account for the missing $30-80$ $\mu \mathrm{m}$ condensation-coalescence bottleneck mechanism of warm rain droplet growth between the upper limit of non-radiating, diffusional condensation growth $(\sim 30 \mu \mathrm{m})$ and the lower limit of turbulent-inertial coalescence growth $(\sim 80 \mu \mathrm{m})$, provided the ambient supersaturation ( $s>0$ ) is not too much depleted by condensation. This amount of radiation effect is also enough to modify significantly the Kohler curves governing equilibrium droplet size distribution. Estimates based on realistic parameters show that radiative transfer, whether with colder upper layers of the atmosphere, warmer lower layers/ground, or solar radiation, can have a significant influence on cloud droplet growth and stability. 


\section{Acknowledgments}

The support of the Hermia G. Soo Professorship and NSF grant 1062361 helped make this research possible.

\section{References}

[1] F. A. Williams, On vaporization of mist by radiation, International Journal of Heat Mass Transfer 8 (1965) 575-587.

[2] S. C. Davies and J. R. Brock, Laser evaporation of droplets, Applied Optics 26 (5) (1987) 786-793.

[3] S. K. Varghese and S. Gangamma, Evaporation of water droplets by radiation: effect of absorbing inclusions, Aerosol and Air Quality Research 7 (1) (2007) 95-105.

[4] G. Miliauskas and V. Sabanas, Interaction of transfer processes during unsteady evaporation of water droplets, International Journal of Heat Mass Transfer 49 (2006) 1790-1803.

[5] C. C. Tseng and R. Viskanta, Enhancement of water droplet evaporation by radiation absorption, Fire Safety Journal 41 (2006) 236-247.

[6] W. M. Kays. M. E. Crawford, and B. Weigand, Convective Heat and Mass Transfer, fourth ed., McGraw-Hill, New York, 2004.

[7] A. F. Mills, Heat and Mass Transfer, Irwin, Chicago, 1995.

[8] K. T. Wang and M. Q. Brewster, Phase-transition radiation in vapor condensation process, International Communications in Heat and Mass Transfer 37 (8) (2010) 945-949.

[9] M. Q. Brewster, Thermal Radiative Transfer and Properties, Wiley, New York, 1992.

[10] M. Q. Brewster, Surface absorption assumption for radiant heating and ignition of energetic solids, Journal of Thermophysics and Heat Transfer, 20 (2) (2006) 348-350.

[11] K. Sassen, Infrared (10.6- $\mu \mathrm{m})$ radiation induced evaporation of large water drops, Journal of the Optical Society of America 71 (7) (1981) 887-891. 
[12] Yu. S., Sedunov, Physics of drop formation in the atmosphere, Halsted/Wiley, New York, 1974, p. 56.

[13] O. Reynolds, On the manner in which raindrops and hailstones are formed. Manchester Literary and Philosophical Society, Memoirs, 3 (6) (1877).

[14] A. R. Stickley, An evaluation of the Bergeron-Findeisen precipitation theory, Monthly Weather Review, October (1940) 272-280.

[15] W. T. Roach, On the effect of radiative exchange on the growth by condensation of a cloud or fog droplet, Quarterly Journal of the Royal Meteorological Society 102 (1976) 361-372.

[16] H. R. Pruppacher and J. D. Klett, Microphysics of Clouds and Precipitation, second ed., Kluwer Academic, New York, 1997.

[17] W. R. Cotton, G. H. Bryan, and S. C. van den Heever, Storm and Cloud Dynamics, second ed., Academic Press/Elsevier, London, 2011.

[18] B. R. Barkstrom, Some effects of 8-12 $\mu \mathrm{m}$ radiant energy transfer on the mass and heat budgets of cloud droplets, Journal of Atmospheric Science 35 (1978) 665-673.

[19] R. Guzzi and R. Rizzi, The effect of radiative exchange on the growth by condensation of a population of droplets, Contributions in Atmospheric Physics 53 (1980) 351-365.

[20] A. Bott, U. Sievers, and W. Zdunkowski, A radiation fog model with detailed treatment of the interaction between radiation and fog microphysics, Journal of Atmospheric Sciences 47 (1990) 2153-2166.

[21] Y. Harrington, G. Feingold, and W. Cotton, Radiative impacts on the growth of a population of drops within simulated summertime Arctic stratus, Journal of Atmospheric Science 57 (2000) 766-785.

[22] W. J. Wiscombe, Scales, tools, and reminiscences, in: A. Marshak and A. B. Davis (Eds.), 3D Radiative Transfer in Cloudy Atmospheres, Springer, Berlin, 2005, pp. 3-92.

[23] P. H. Austin, S. Siems, and Y. Wang, Constraints on droplet growth in radiatively cooled stratocumulus clouds, Journal of Geophysical Research 100 (1995) $14,231-14,242$.

[24] W. J. Wiscombe, R. M. Welch, and W. D. Hall, The effects of very large drops on cloud absorption, Part I: Parcel models, Journal of Atmospheric Science 41 (1984) 1336-1355. 
[25] C. Hartman and Y. Harrington, Radiative impacts on the growth of drops within simulated marine stratocumulus. Part I: maximum solar heating, Journal of Atmospheric Science 62 (2005) 2323-2338.

[26] J. Marquis and Y. Harrington, Radiative influences on drop and cloud condensation nuclei equilibrium in stratocumulus, Journal of Geophysical Research 110 (2005) doi:10.1029/2004JD005401.

[27] L. Dufour and R. Defay, Thermodynamics of clouds, Academic Press, New York, 1963, pp. 91-95. 


\section{List of Figures}

1. Thermodynamic state definitions and energy fluxes at water vapor-liquid interface for (a) spherical problem (arbitrary droplet) and (b) Cartesian problem (large droplet).

2. Volumetric absorption coefficient, $\mathrm{K}_{\mathrm{a}}$, of liquid water at room temperature [9].

3. Temperature profile in liquid phase for semi-infinite (opaque) water subject to radiant flux of $1070 \mathrm{~W} / \mathrm{m}^{2}$ with $T_{\mathrm{s}}=\mathrm{T}_{\mathrm{e}}=22^{\circ} \mathrm{C}$ (QS, adiabatic liquid), surface regression speed $0.43 \mu \mathrm{m} / \mathrm{s}$, zero interface absorption $\left(f_{s}=0\right)$, and with $1 / \mathrm{K}_{\mathrm{a}}(=$ $\delta_{\mathrm{r}}$ ) as a parameter. Advective term is both included (solid lines, $\delta_{\mathrm{t}}=32.7 \mathrm{~cm}$ ) and neglected (dashed lines, $\delta_{\mathrm{t}} \rightarrow \infty$ ). Gas-phase parameters are as yet unspecified.

4. Same as Fig. 3 for the mid-IR absorption case $\left(1 / K_{a}=20 \mu \mathrm{m}\right)$, showing detail near interface (solid and dashed lines are indistinguishable).

5. Gas- and liquid-phase temperature profiles for $\mathrm{T}_{e}=22^{\circ} \mathrm{C}, \mathrm{RH}=25 \%, \mathrm{P}=86$ $\mathrm{kPa}, \mathrm{L}=1 \mathrm{~mm}$, and an optically thick, $\mathrm{QS}$ adiabatic liquid phase with $1 / \mathrm{K}_{\mathrm{a}}=0\left(\mathrm{f}_{\mathrm{s}}\right.$ $=1)$ or $10 \mathrm{~cm}\left(f_{s}=0\right)$. This makes conditions for $1070 \mathrm{~W} / \mathrm{m}^{2}\left(f_{s}=0\right)$ the same as those for the $10-\mathrm{cm}\left(\mathrm{f}_{\mathrm{s}}=0\right)$ case of Fig. 3 .

6. Cartesian solution for heat fluxes and $T_{s}$ vs. radiant flux for same conditions as Fig. 5 (also applies to an optically thick, adiabatic droplet of $1 \mathrm{~mm}$ radius).

7. Internal temperature for QS adiabatic droplet neglecting advective term: (a) radial profile for various droplet sizes with $1 / \mathrm{K}_{\mathrm{a}}=20 \mu \mathrm{m}$ (mid-IR) and (b) maximum change for $R=100 \mu \mathrm{m}, \mathrm{q}_{\mathrm{r}}=5000 \mathrm{~W} / \mathrm{m}^{2}$ over VIS/IR spectrum.

8. Comparison of theory with measurement [11] for droplet (a) temperature and (b) radius versus time for $\mathrm{CO}_{2}$ laser irradiated droplet with both unsteady and QS behavior.

9. Effect of radiation on cloud droplet growth rate for hypothetical droplet near the top of a cloud with conditions representative of lower troposphere, tropical to midlatitude summertime: $20^{\circ} \mathrm{C}, 70 \mathrm{kPa}$. Radiation is to a colder $0^{\circ} \mathrm{C}$ upper atmospheric layer (e.g., cloud) with radiation parameter $\mathcal{Q}_{\mathrm{r}}=-5.5 \mathrm{e}-5 \mu \mathrm{m}^{-1}$. A slightly supersaturated environment is assumed, $s=0.1 \%$.

10. Same as Fig. 9 except slightly subsaturated environment, $s=-0.1 \%$.

11. Equilibrium supersaturation vs. cloud droplet diameter (Kelvin-radiation Kohler curves) for conditions similar to Figs. 9 and 10. 
Fig. 1

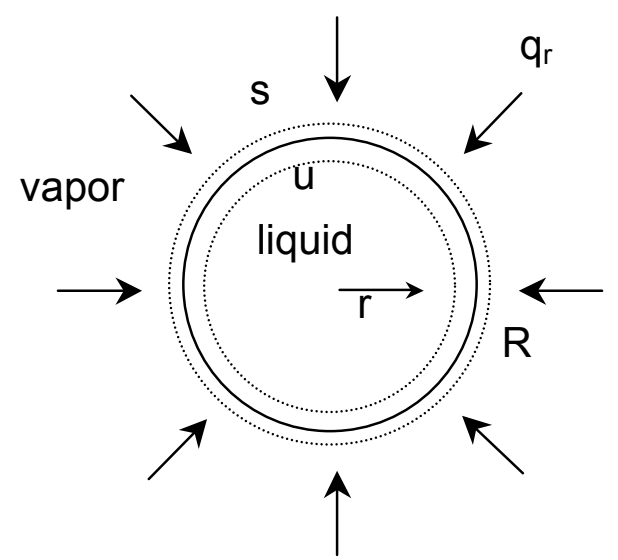

(a)

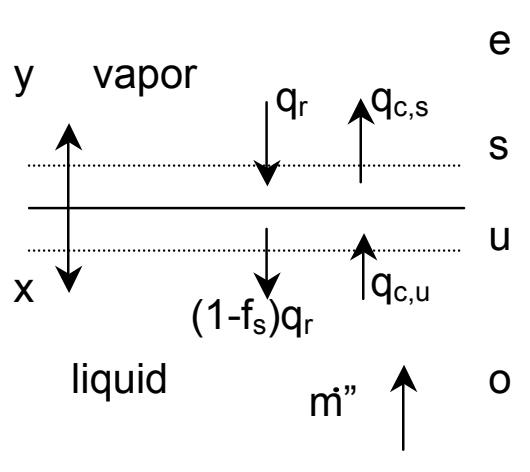

(b) 
Fig. 2

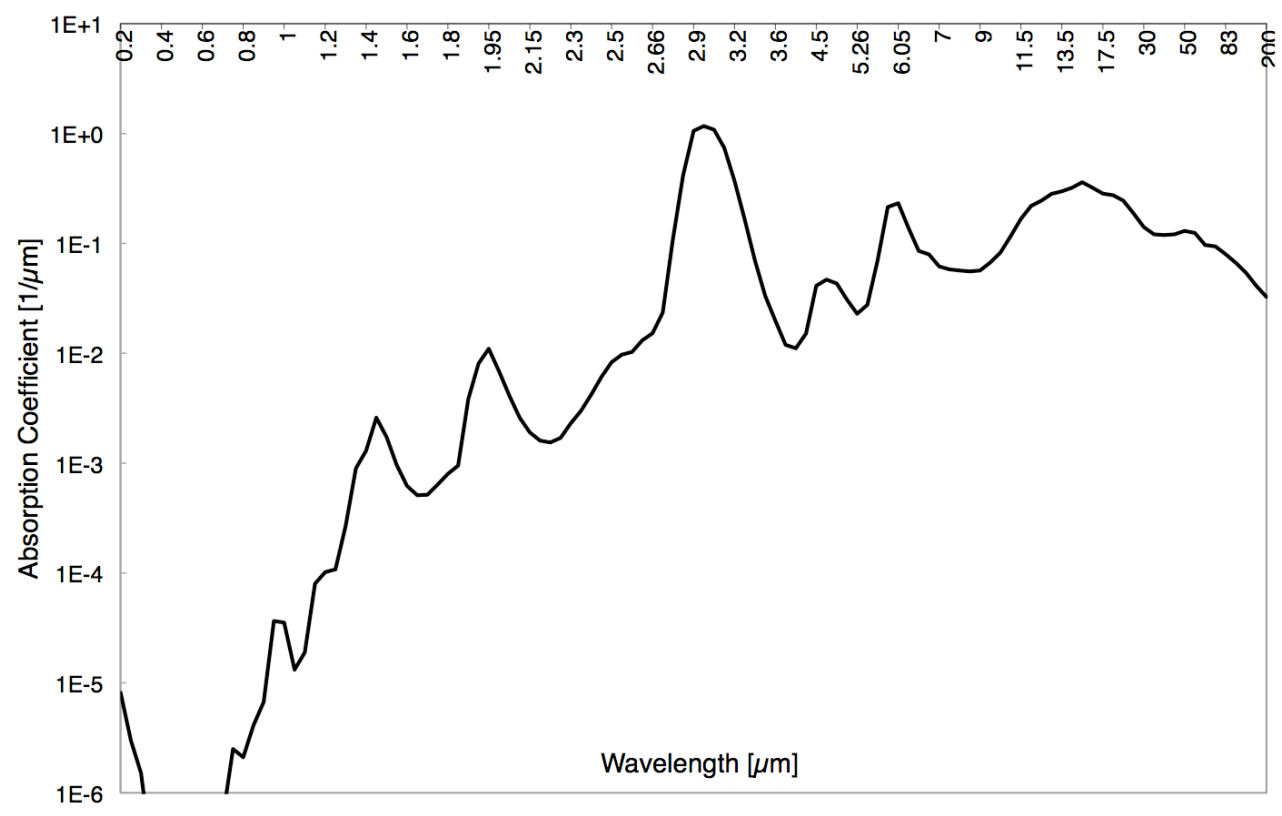


Fig. 3

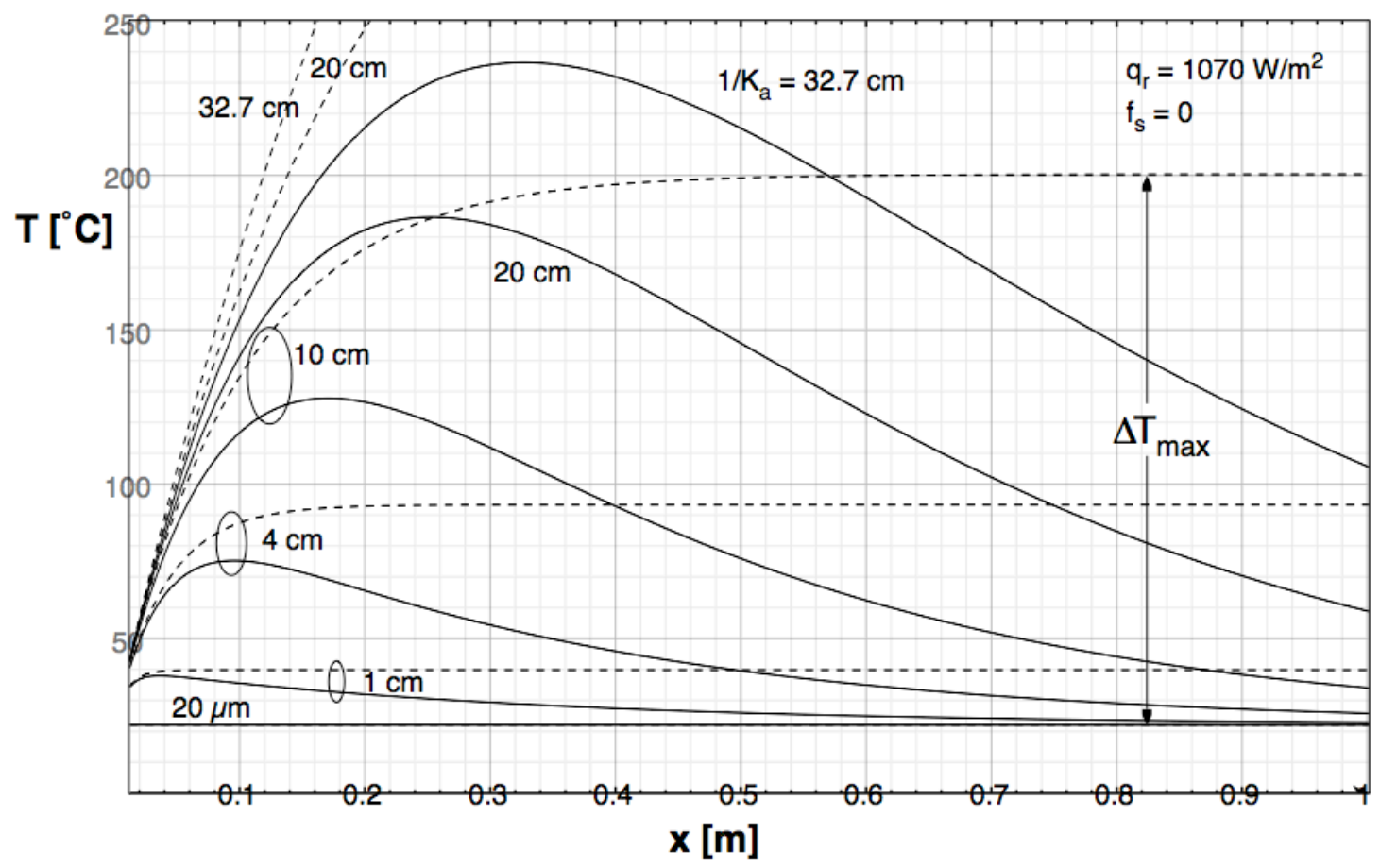


Fig. 4

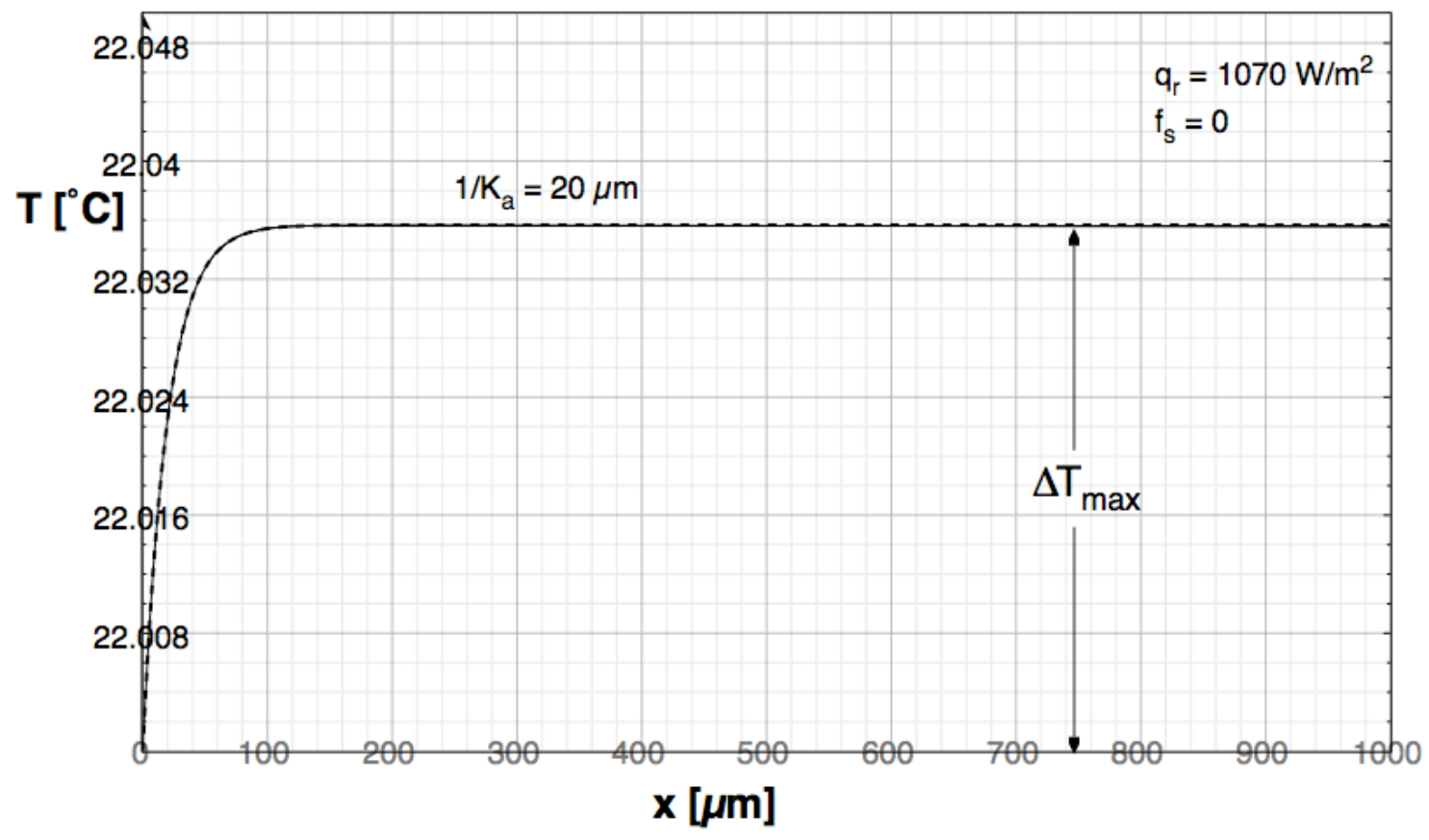


Fig. 5

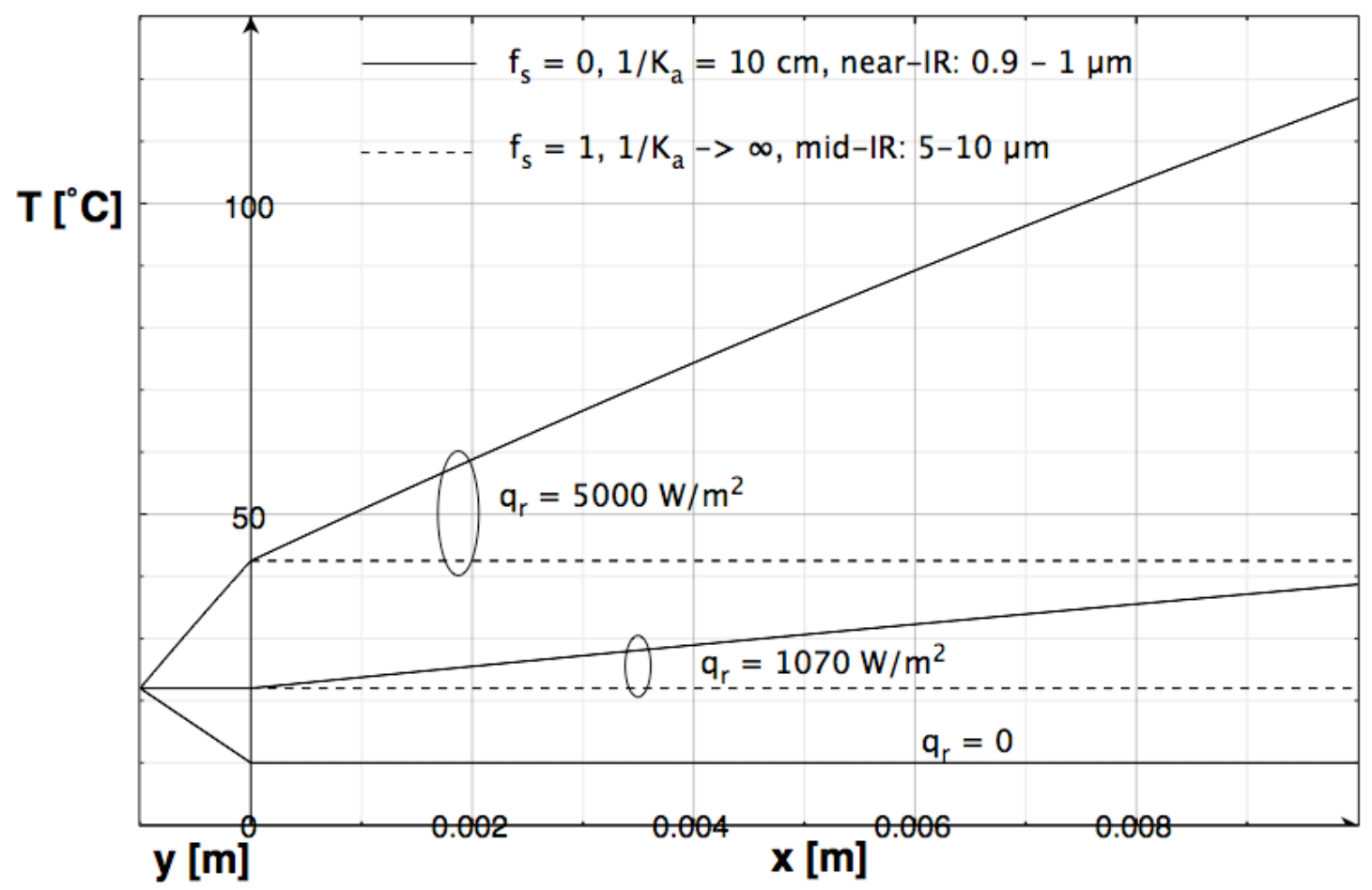


Fig. 6

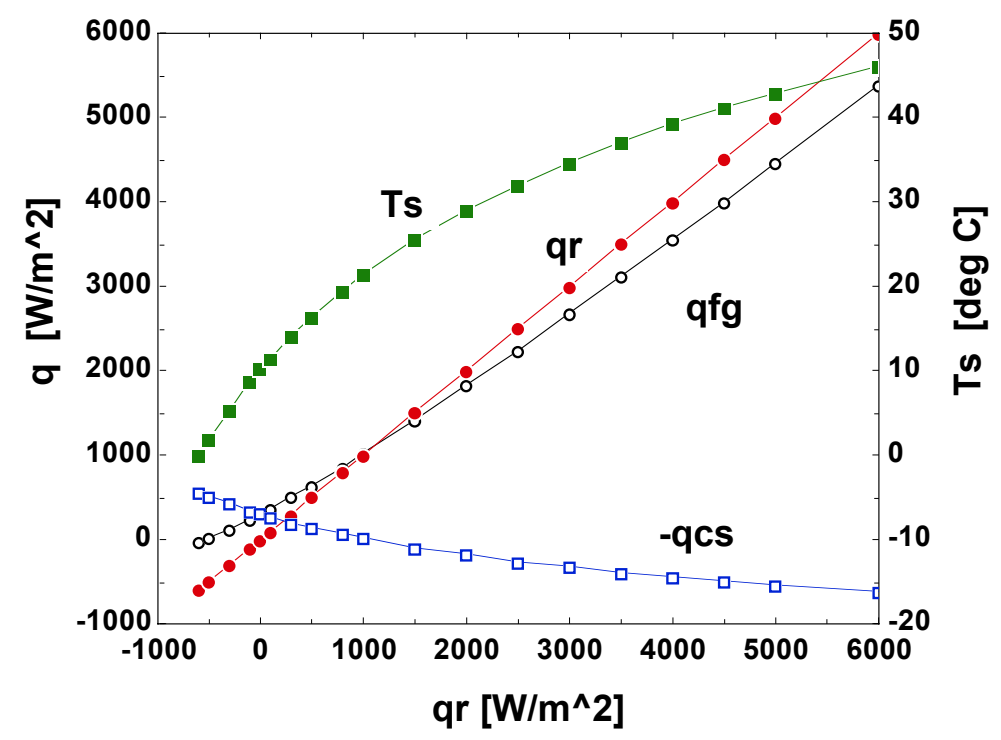


Fig. 7

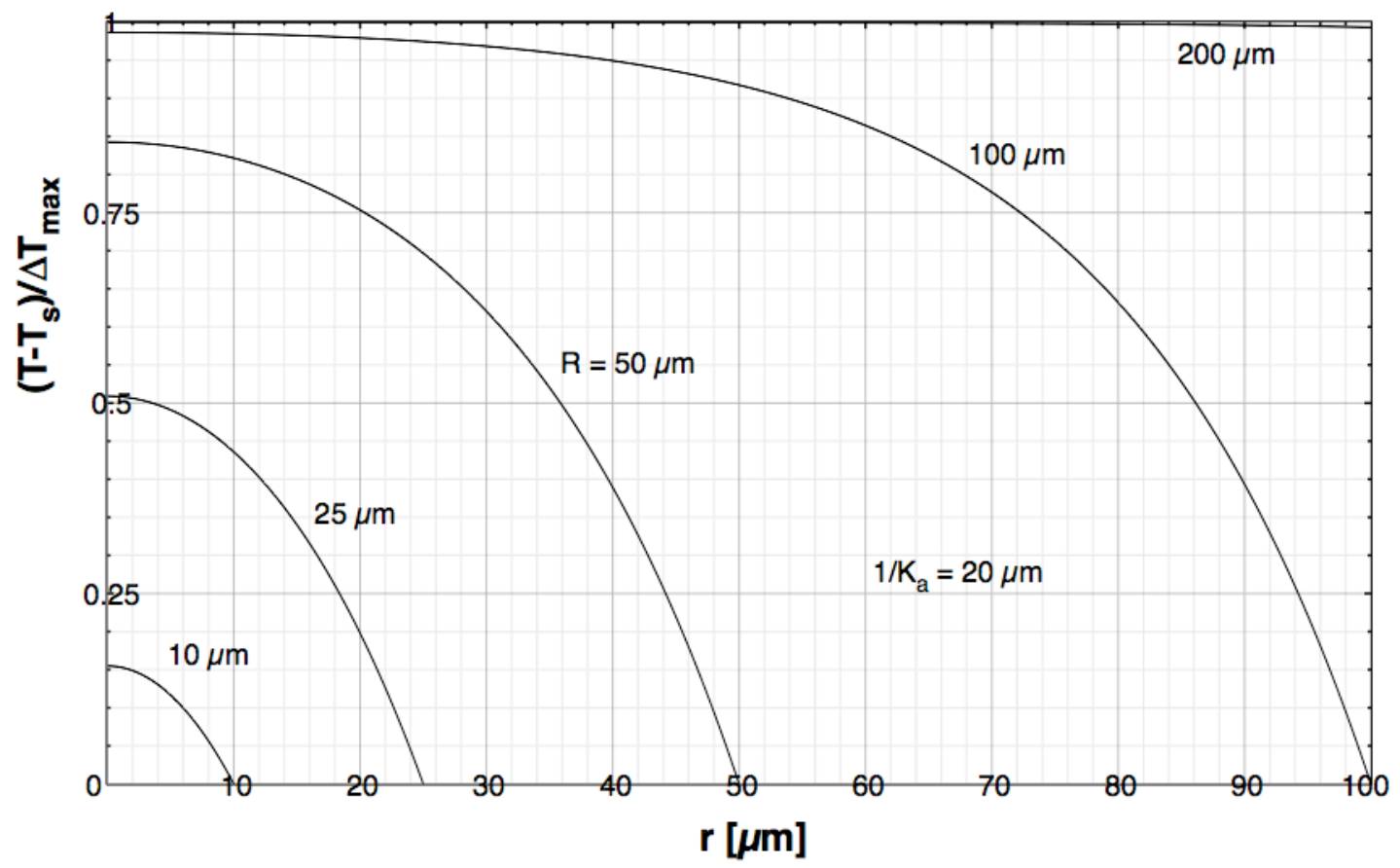

(a)

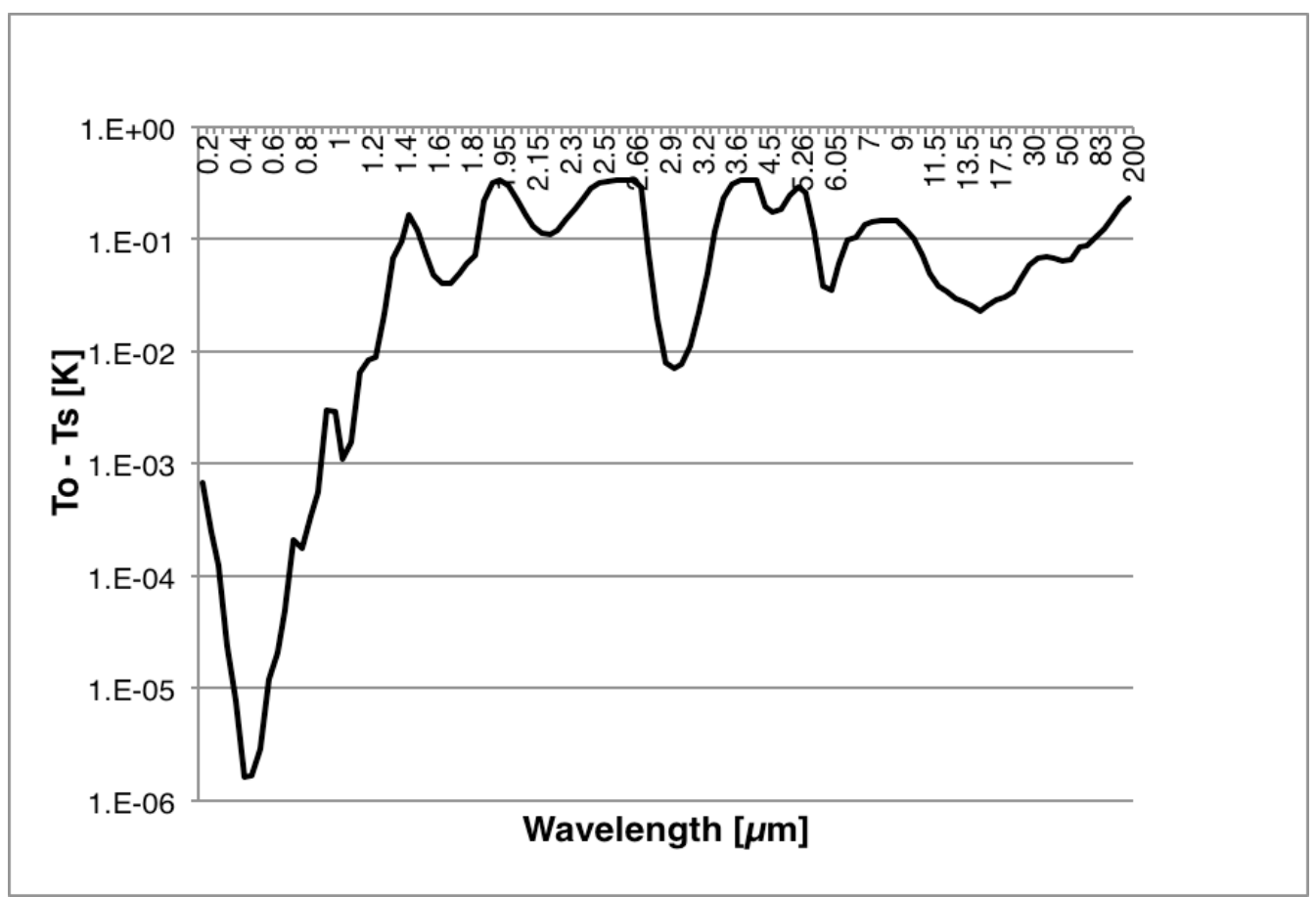

(b) 
Fig. 8

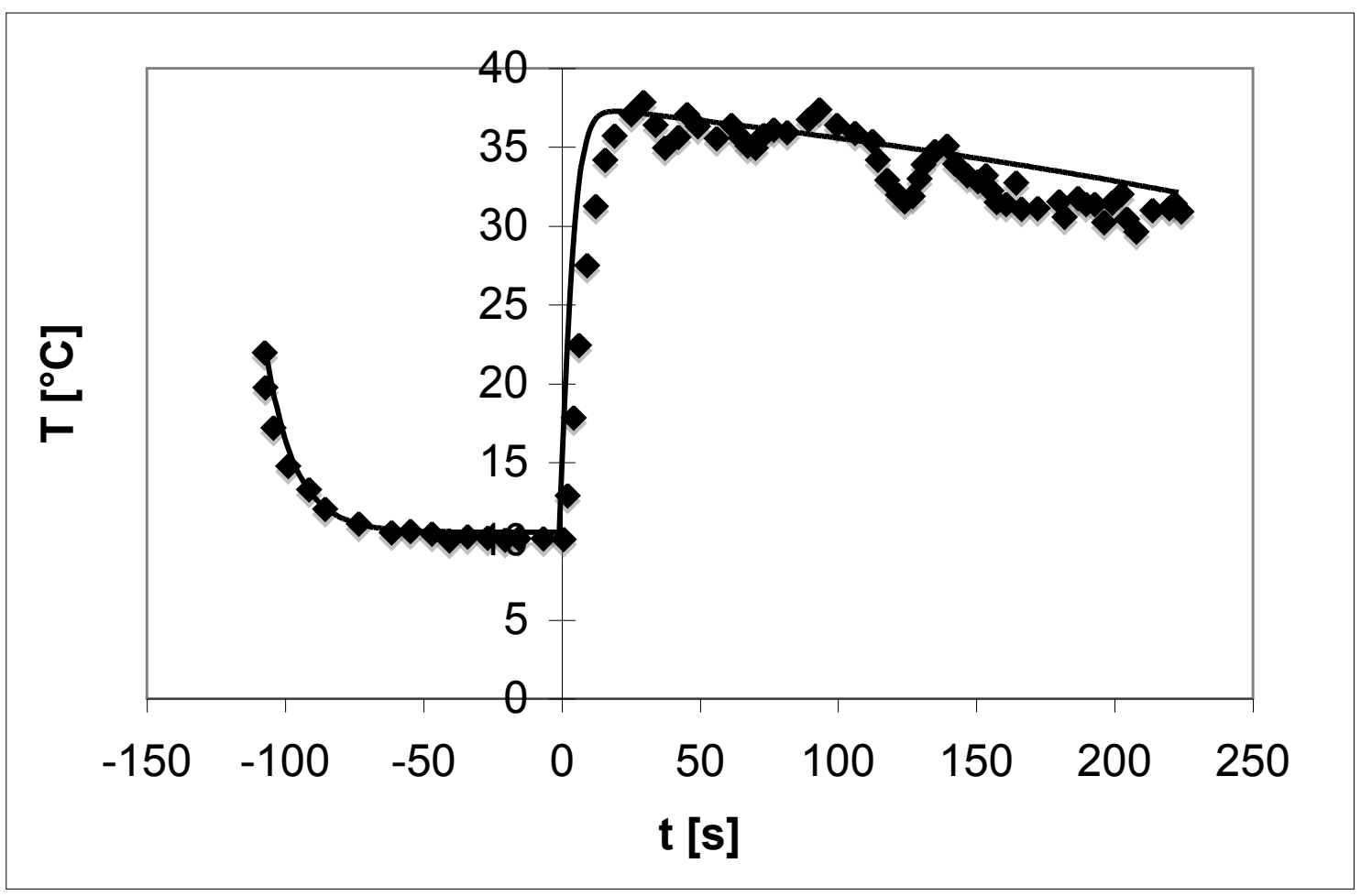

(a)

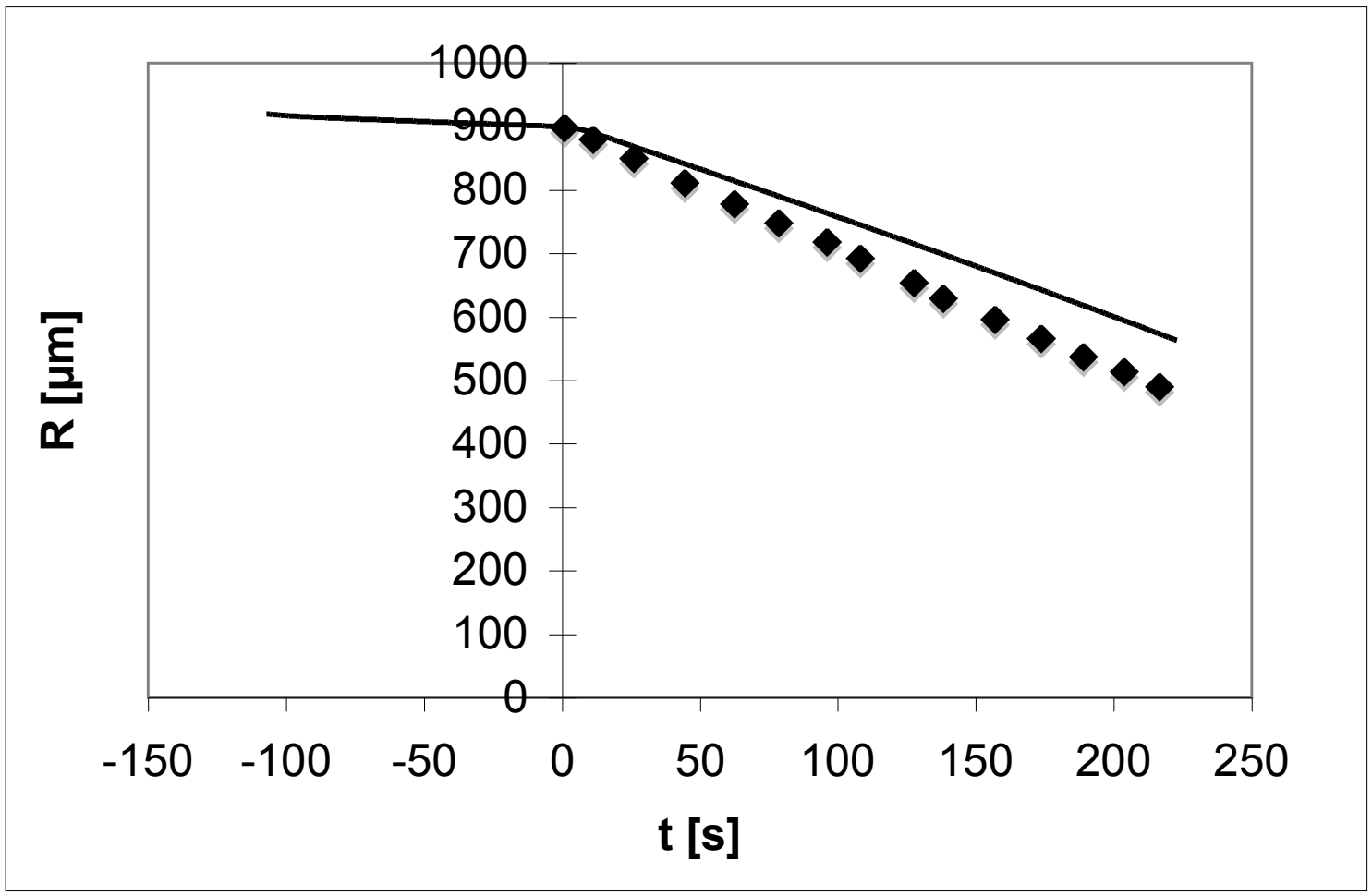

(b) 
Fig. 9

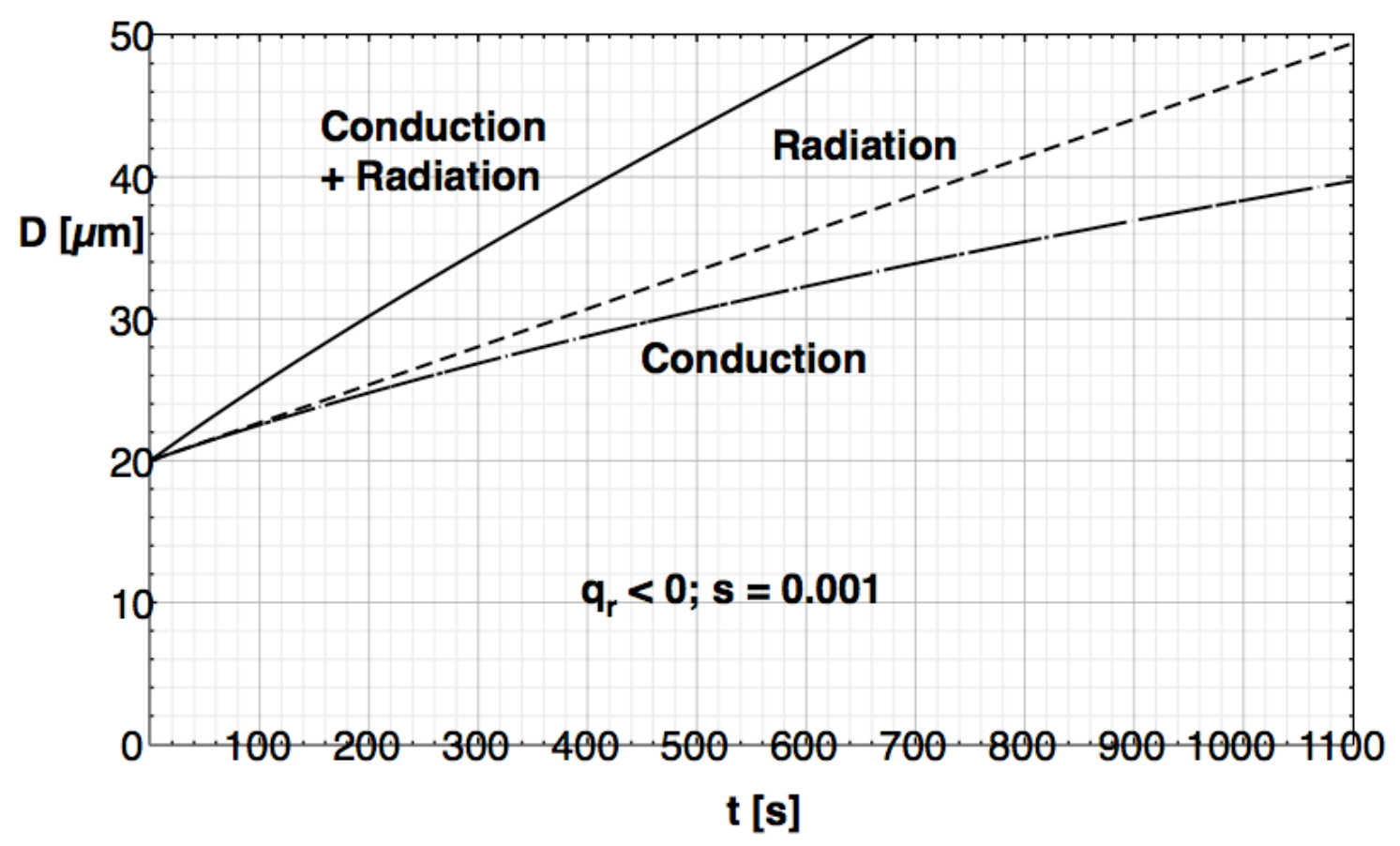


Fig. 10

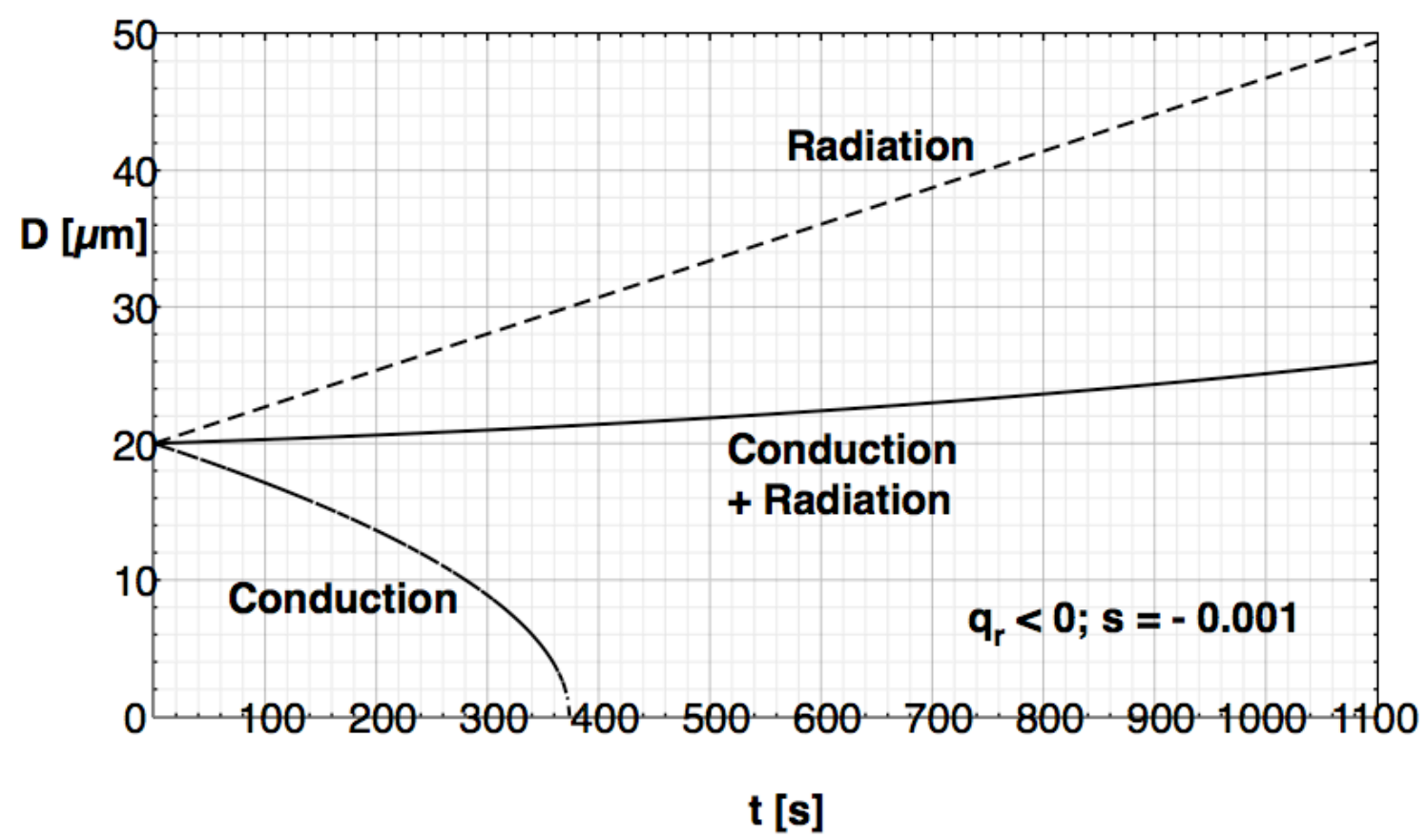


Fig. 11

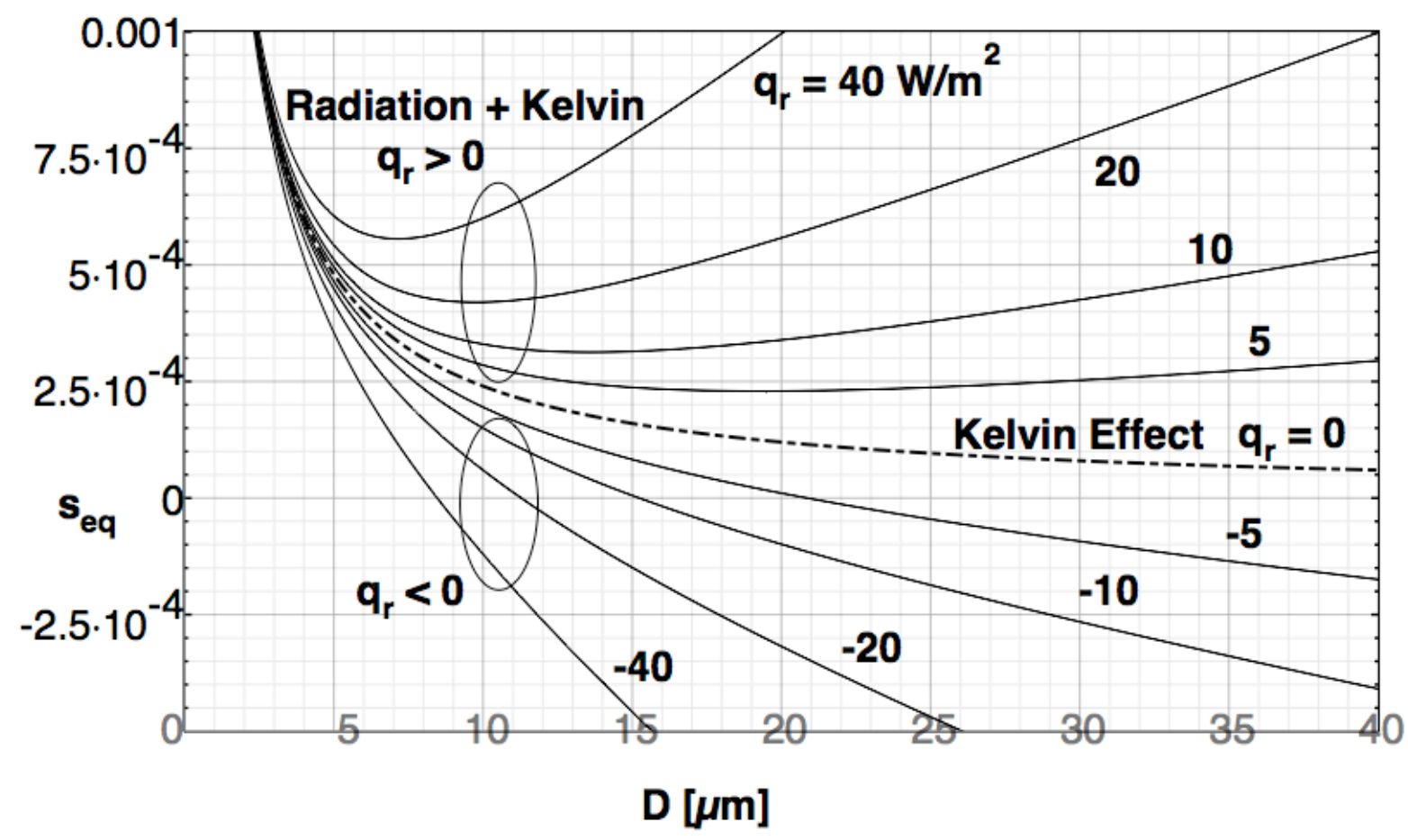

